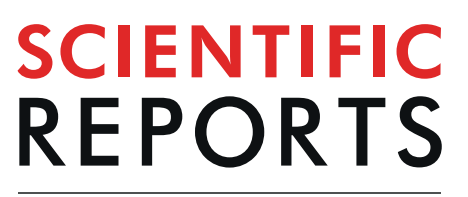

natureresearch

\title{
Combined behavioral and electrophysiological evidence for a direct cortical effect of prefrontal tDCS on disorders of consciousness
}

\author{
Bertrand Hermann $\mathbb{1}^{1,2,3,4}$, Federico Raimondo ${ }^{1,2,3,5,6}$, Lukas Hirsch ${ }^{7}$, Yu Huang ${ }^{7}$, \\ Mélanie Denis-Valente ${ }^{1,2,3,8}$, Pauline Pérez ${ }^{1,2,3}$, Denis Engemann ${ }^{9,10}{ }^{9}$, Frédéric Faugeras ${ }^{1,2,3}$, \\ Nicolas Weiss $\mathbb{1}^{4,11}$, Sophie Demeret ${ }^{4}$, Benjamin Rohaut ${ }^{1,2,3,12}$, Lucas C. Parra $\mathbb{1}^{7}$, \\ Jacobo D. Sitt ${ }^{1,2,3,13^{*}}$ \& Lionel Naccache ${ }^{1,2,3,8,11,13^{*}}$
}

Severe brain injuries can lead to long-lasting disorders of consciousness (DoC) such as vegetative state/ unresponsive wakefulness syndrome (VS/UWS) or minimally conscious state (MCS). While behavioral assessment remains the gold standard to determine conscious state, EEG has proven to be a promising complementary tool to monitor the effect of new therapeutics. Encouraging results have been obtained with invasive electrical stimulation of the brain, and recent studies identified transcranial direct current stimulation (tDCS) as an effective approach in randomized controlled trials. This non-invasive and inexpensive tool may turn out to be the preferred treatment option. However, its mechanisms of action and physiological effects on brain activity remain unclear and debated. Here, we stimulated 60 DoC patients with the anode placed over left-dorsolateral prefrontal cortex in a prospective openlabel study. Clinical behavioral assessment improved in twelve patients (20\%) and none deteriorated. This behavioral response after tDCS coincided with an enhancement of putative EEG markers of consciousness: in comparison with non-responders, responders showed increases of power and longrange cortico-cortical functional connectivity in the theta-alpha band, and a larger and more sustained P300 suggesting improved conscious access to auditory novelty. The EEG changes correlated with electric fields strengths in prefrontal cortices, and no correlation was found on the scalp. Taken together, this prospective intervention in a large cohort of DoC patients strengthens the validity of the proposed EEG signatures of consciousness, and is suggestive of a direct causal effect of tDCS on consciousness.

After an acute phase of coma, severe acute brain injuries can lead to lasting disorders of consciousness (DoC). The Coma Recovery Scale-Revised (CRS-R) $)^{1,2}$ is the most widely accepted tool to distinguish vegetative state/ unresponsive wakefulness syndrome (VS/UWS) from minimally conscious state (MCS) patients. While VS/UWS only show non-purposeful reflexive behaviors, MCS show reproducible yet inconsistent cognitive and intentional cortically-mediated behaviors ${ }^{3}$. Finally, exit-MCS patients exhibit functional communication or use of objects. However, this behavioral assessment has limitations and $15-20 \%$ of behaviorally-diagnosed VS/UWS patients

\footnotetext{
${ }^{1}$ Institut du Cerveau et de la Moelle épinière, ICM, F-75013, Paris, France. ${ }^{2}$ Inserm U 1127, F-75013, Paris, France. ${ }^{3}$ CNRS UMR 7225, F-75013, Paris, France. ${ }^{4}$ Department of Neurology, Neuro ICU, Hôpital de la Pitié-Salpêtrière, AP-HP, F-75013, Paris, France. ${ }^{5}$ Laboratorio de Inteligencia Artificial Aplicada, Departamento de Computación FCEyN, UBA, C1428EGA, Ciudad Autónoma de Buenos Aires, Argentina. ${ }^{6}$ CONICET - Universidad de Buenos Aires, Instituto de Investigación en Ciencias de la Computación, Godoy Cruz 2290, C1425FOB, Ciudad Autónoma de Buenos Aires, Argentina. ${ }^{7}$ Biomedical Engineering Department, City College of the City University of New York, New York, NY, 10031, USA. ${ }^{8}$ Department of Neurophysiology, Hôpital de la Pitié-Salpêtrière, AP-HP, F-75013, Paris, France. ${ }^{9}$ Parietal project-team, INRIA, Université Paris-Saclay, 91120, Saclay, France. ${ }^{10}$ Cognitive Neuroimaging Unit, CEA DSV/I2BM, INSERM, Université Paris-Sud, Université Paris-Saclay, NeuroSpin center, 91191, Gif-sur-Yvette, France. ${ }^{11}$ Faculté de Médecine Pitié-Salpêtrière, Sorbonne Université, 75013, Paris, France. ${ }^{12}$ Department of Neurology, Columbia University, New York, NY, 10027, USA. ${ }^{13}$ These authors contributed equally: Jacobo D. Sitt and Lionel Naccache.*email: jacobo.sitt@icm-institute.org; lionel.naccache@gmail.com
} 
A

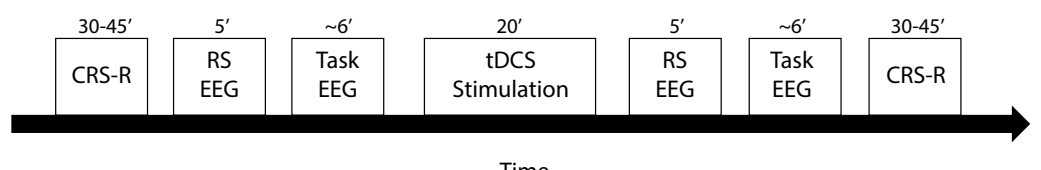

Time

B

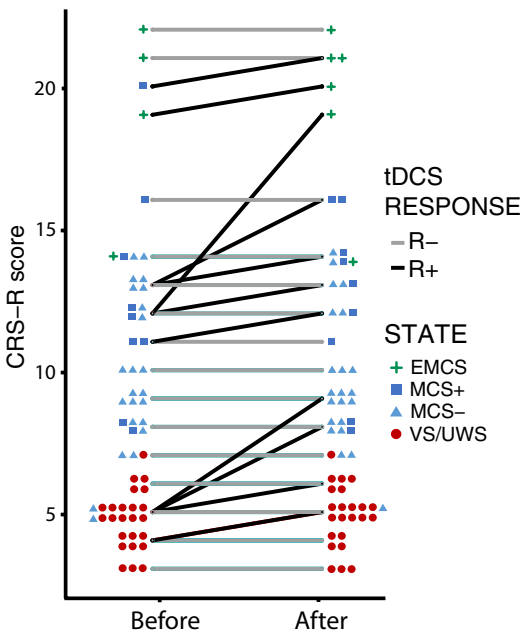

C

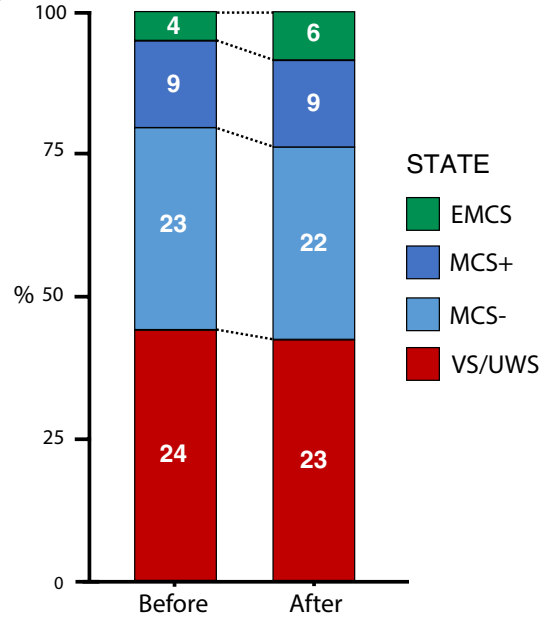

Figure 1. Behavioral response to tDCS. (A) Study protocol timeline showing the behavioral (Coma Recovery Scale-revised - CRS-R-) and electrophysiological (i.e. resting-state (RS) and auditory oddball paradigm (task-EEG)) measures of the effects consecutive to a single transcranial direct current stimulation (tDCS) session. (B) Individual patients' CRS-R scores before and after $\mathrm{tDCS}$ are represented for responders $(\mathrm{R}+$, in black) and non-responders (R-, in gray), together with the number of patients and their state (symbols). (C) The proportion of each state of consciousness, before and after tDCS showed an increase in the higher states of consciousness (exit minimally conscious state (EMCS) and minimally conscious state 'plus' (MCS + )), at the expense of lower states of consciousness (vegetative state/unresponsive wakefulness syndrome (VS/UWS) and MCS 'minus' (MCS-)).

show patterns of brain activity suggestive of higher states of consciousness ${ }^{4-8}$. Among the different brain-imaging techniques used, EEG has proved to be a reliable, non-invasive bedside tool to probe signatures of both conscious state and conscious access to external stimuli in DoC patients ${ }^{9-15}$. Specifically, increases of spectral power, complexity and functional connectivity in the theta-alpha bands correlate with state of consciousness ${ }^{11,13,16}$, but the specificity and causal value of these putative signatures of consciousness remain to be demonstrated. Nevertheless, the combination of such behavioral and EEG measures seems optimal to assess in detail possible improvements of consciousness during treatment with new therapeutic techniques.

Encouraging results have been obtained in DoC patients with invasive electrical stimulation of the brain ${ }^{17-20}$. Recently, transcranial delivery of a low intensity electrical current over the scalp with transcranial direct current stimulation (tDCS) has shown potential benefit in improving conscious state of DoC patients as assessed by the CRS- $\mathrm{R}^{21-27}$. However, some authors did not find this improvement of consciousness following tDCS ${ }^{28-32}$. More generally the efficacy of tDCS is debated ${ }^{33-39}$. This skepticism is mostly due to the fact that its mechanisms have not been firmly established yet and that most studies reporting behavioral results did not investigate the effects of tDCS on brain activity. Specifically in DoC patients, the effects of tDCS on brain activity have only been investigated in a few small sample studies using sparse and diverse EEG metrics, roughly showing an increase in fronto-parietal coherence in high frequencies ${ }^{29,30}$, decrease in lower frequencies ${ }^{26}$ and increase in cortical excitability following $\mathrm{tDCS}^{28,40}$. This diversity of metrics and the lack of clear underlying hypotheses regarding the electrophysiology of consciousness make it hard to interpret the effect of tDCS on consciousness. As a result of both these behavioral and electrophysiological findings, tDCS is not recommended for the treatment of disorders of consciousness ${ }^{41}$.

In this prospective case-control open-label study, we evaluated the impact of prefrontal tDCS (Fig. 1A) on both the behavior and hypothesis-driven quantitative electrophysiological measures of consciousness, by combining for the first time measures of the two complementary key aspects of consciousness ${ }^{42}$ : conscious state (irrespective of the current conscious content), and conscious access to a specific content in the context of an auditory task. We made the hypothesis that behavioral improvement after tDCS would be paralleled by an improvement of these comprehensive electrophysiological signatures of consciousness, that is the aforementioned putative EEG markers of conscious access but also the P300 cognitive evoked response potential correlate of conscious access to external stimuli $^{43-46}$. We then investigated the possible mechanism of action of $\mathrm{tDCS}$ by correlating the electrophysiological response to the applied electric fields estimated from individual patients' head and brain MRI anatomy.

\section{Results}

Behavioral response after one tDCS session. Between October 2015 and September 2018, among 69 eligible DoC patients, 66 patients were treated prospectively with a single 20 minutes tDCS session with the anode placed over the left dorsolateral prefrontal cortex and the cathode over the right supraorbital cortex, using the 
same parameters as previously reported in DoC patients ${ }^{22}$. The effects of this tDCS session were evaluated by a combined behavioral and electrophysiological assessment using CRS-R and high-density EEG recordings at rest and during an auditory oddball paradigm immediately before and after the stimulation (Fig. 1). Among these, 60 patients could be included in the study (one was comatose, one had a seizure during EEG and four had insufficient EEG data for subsequent analyses, SI section B1, Fig. S1). The cohort was thus composed of $24 \mathrm{VS} / \mathrm{UWS}, 32 \mathrm{MCS}$ and 4 exit-MCS patients (SI section B1, Table S2). Response to tDCS (R+), defined as an increase of the CRS-R score after stimulation as compared to before, was observed in 12 patients (20\%): 4 VS/ UWS (16.7\%), 7 MCS (21.9\%) and 1 exit-MCS patients (25\%), without differences across groups ( $p>0.8$, Fisher's exact test). This resulted in a change in conscious state in 3 patients, one VS/UWS shifting towards MCS and two MCS shifting towards exit-MCS (Fig. 1B,C). This proportion of $\mathrm{R}+$ is close to the $27 \%$ responders reported in a double-blind randomized trial with the same stimulation parameters ${ }^{22}$. Conversely, non-responders $(\mathrm{R}-)$ were defined by CRS-R scores that were either stable or decreasing after $\mathrm{tDCS}$. Interestingly, none of the patients showed a decrease of CRS-R score. Overall, CRS-R score changes after stimulation were significantly larger than zero $(p=0.002$, Wilcoxon signed-rank test, effect size $r=0.28$ [0.21-0.36]). No significant differences were found between $\mathrm{R}+$ and $\mathrm{R}-$ populations in their demographic characteristics (age, sex, etiology and time since injury), and, importantly, neither in their vigilance before tDCS, nor data preprocessing (SI section B1, Table S3). No adverse effect was observed.

Spectral power and connectivity in the theta-alpha band increase in responders to tDCS. We first analyzed the interaction between resting-state brain activity and the behavioral response after tDCS stimulation using 5-minutes of EEG acquired before and after stimulation. To that end, we computed putative signatures of conscious state on both recordings using an automated procedure reported previously ${ }^{13,15,47}$ (see Methods). We then tested whether these EEG signatures were modified by tDCS differently in $\mathrm{R}+$ than in $\mathrm{R}-$, using the following contrast: [post - pre] in $\mathrm{R}+>$ [post - pre] in $\mathrm{R}$ - with multiple comparisons correction through permutation-based cluster analysis (see Statistical analysis in Methods section).

Power spectral density. After stimulation, as compared to $\mathrm{R}-, \mathrm{R}+$ showed a significant increase in normalized theta power with a topography maximal over the parietal cortices (cluster $p=0.03$, effect size $g=0.92[0.56-2.03]$; Fig. 2). Similarly, we observed an increase of both raw and normalized alpha power (cluster $p=0.01, g=0.80$ [0.44-0.80] and cluster $p=0.04, g=1.10[0.79-1.91]$ respectively). Other markers of spectral power did not differ between $\mathrm{R}-$ and $\mathrm{R}+$ (SI section B2, Fig. S2).

Complexity. EEG complexity, assessed by the permutation entropy in the theta-alpha band, showed a trend of an increase in $\mathrm{R}+$ as compared to $\mathrm{R}-$ in the same parietal region (cluster $p=0.07, g=0.70[-0.11-1.05]$ ). Kolmogorov and spectral entropy did not differ.

Functional connectivity. Response to tDCS was also characterized by an increase of functional connectivity in the theta-alpha band $(4-10 \mathrm{~Hz})$, assessed by the weighted symbolic mutual information, which captures both linear and non-linear coupling between pairs of electrodes and was previously shown to scale with conscious state ${ }^{11}$. When comparing topographies of averaged values, we found a parieto-occipital cluster with increased values in $\mathrm{R}+$ as compared to $\mathrm{R}-$ patients $(p=0.01, g=0.82[0.11-1.62]$ ) (Fig. 2, last row). This was confirmed by the analysis conducted across all pairs of electrodes: 4 significant clusters of electrodes, located over a seemingly single centro-parietal hub encompassing parietal and occipital cortices, showed larger values of functional connectivity for $\mathrm{R}+$ than for $\mathrm{R}$ - patients (respective $\mathrm{p}$-values were $0.01,0.02,0.03$ and 0.04 with a global $g=1.27$ [0.52-1.54]; Fig. 3A and SI section B3, Fig. S3). These differences between $\mathrm{R}+$ and $\mathrm{R}-$ were explained by an increase in $\mathrm{R}+$ patients after stimulation as compared to before (cluster $p=0.02, g=0.81$ [0.54-1.21]), while no significant change was found in $\mathrm{R}-$ patients (Fig. $3 \mathrm{~B}$ ). We found no differences between $\mathrm{R}+$ and $\mathrm{R}-$ in both the functional connectivity in the delta-theta band $(2-5 \mathrm{~Hz})$ and in the alpha-beta band $(8-20 \mathrm{~Hz})$ (data not shown). Note that tDCS effects did not appear systematically different for any of these markers when comparing VS/UWS patients to MCS and exit-MCS patients (SI section B4, Fig. S4).

A neural signature of conscious access improves in responders to tDCS. In addition to resting-state, we assessed the impact of tDCS on the ability of patients to detect auditory regularities during an oddball paradigm (modified from ${ }^{48}$, a task known to require conscious access to auditory novelty). Patients were instructed to actively count the occurrence of auditory oddballs (series of 4 identical tones followed by a $5^{\text {th }}$ distinct tone; $20 \%$ of trials) delivered randomly among series of 5 identical tones (standard trials; $80 \%$ of trials). We computed the event-related potentials (ERP) to deviant tone minus standard tone, before and after tDCS, and compared $\mathrm{R}+$ with $\mathrm{R}$ - using the same interaction contrast as for the resting-state. Five EEG recordings were discarded after automatic assessment of data quality. The analysis performed on the 55 remaining datasets $(11 \mathrm{R}+$ and $44 \mathrm{R}-$ ) revealed a significant positive and left-lateralized anterior cluster spanning from $28 \mathrm{~ms}$ to $376 \mathrm{~ms}$ after the onset of the $5^{\text {th }}$ sound $(p=0.008, g=1.40$ [0.84-1.93], Fig. 4A). Pre/post comparisons localized the origin of this effect to two significant clusters in $\mathrm{R}+$ patient (a first posterior cluster from $52-312 \mathrm{~ms}, p=0.03, g=-0.96$ $[-1.52-0.38]$ and a second left-lateralized anterior cluster from $68-392 \mathrm{~ms}, p=0.02 ; g=1.26[0.78-1.84])$. Although the ERP response observed after stimulation started early after the $5^{\text {th }}$ sound it was sustained in time and peaked around $200 \mathrm{~ms}$ as a typical P3a component ${ }^{49,50}$, indicative of high-level processing of auditory novelty. By contrast, only a classical mismatch negativity ${ }^{51,52}$ was observed in $\mathrm{R}+$ before stimulation and no difference was observed in R- patients (Fig. 4B and SI section B6, Fig. S6A).

We supplemented this univariate analysis with a multivariate temporal generalization decoding $\operatorname{method}^{53} \mathrm{cou}^{-}$ pled with cluster-based permutation analysis in order to better characterize the dynamics of ERP independently 
Averaged Measures

$\mathrm{R}-$ (After>Before) (After $>$ Before)

$|\delta|$

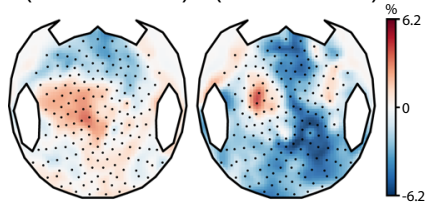

$|\theta|$

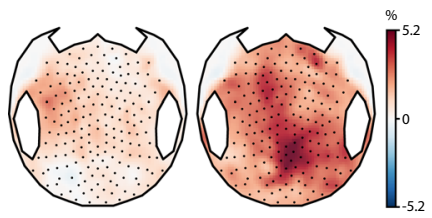

$|a|$

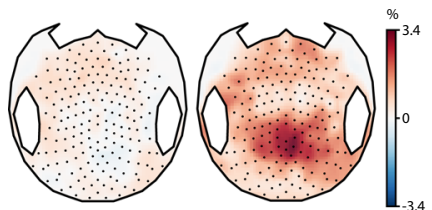

$|\beta|$

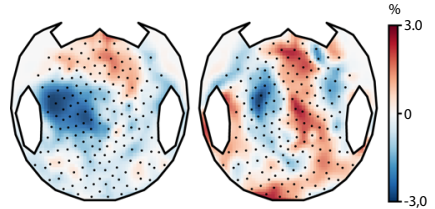

$|\gamma|$
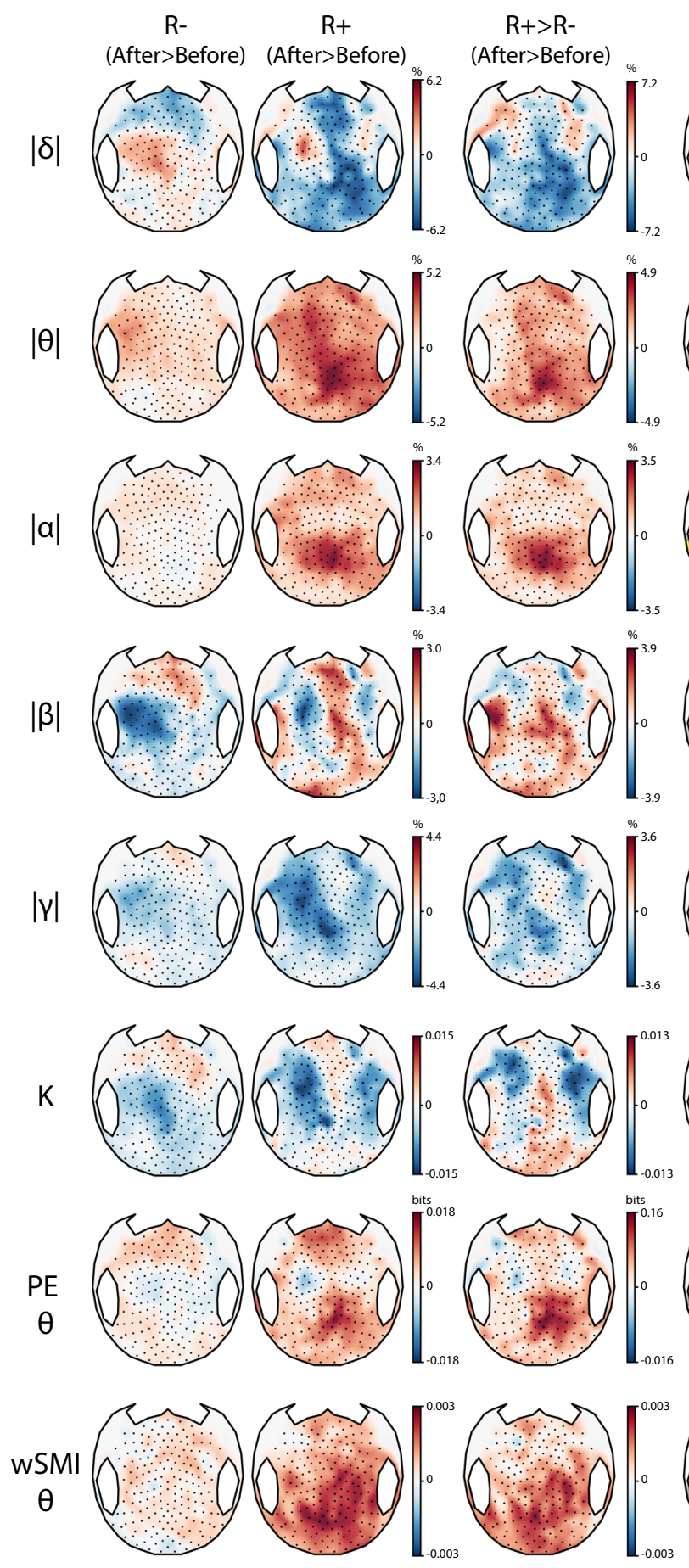

Statistics
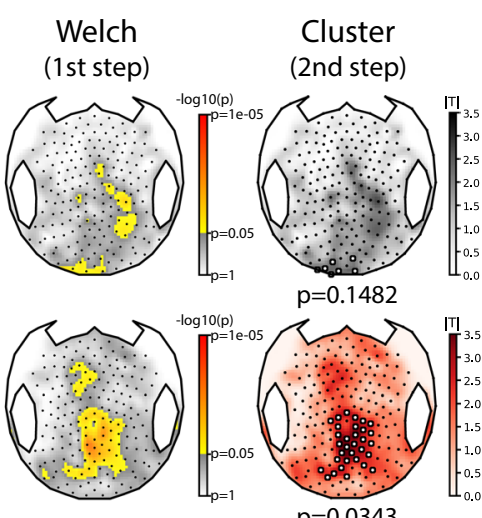

$\mathrm{p}=0.1482$
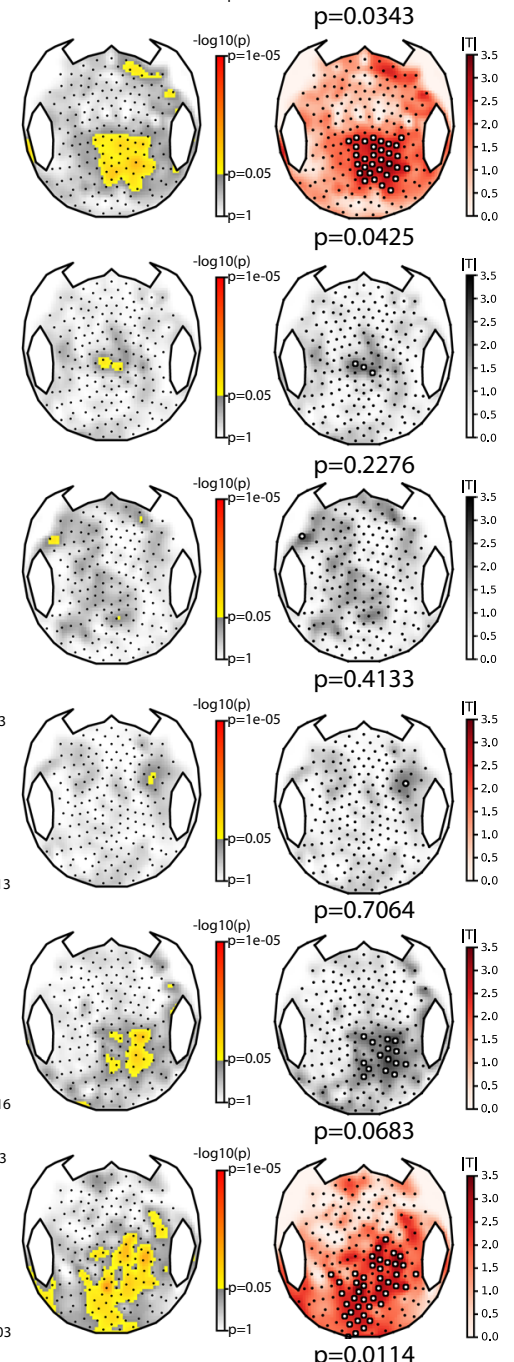

Figure 2. Resting-state EEG markers increases after tDCS in responders. Topographic representations of the tDCS-induced changes in normalized spectral power (delta $|\delta|$, theta $|\theta|$, alpha $|\alpha|$, beta $|\beta|$ and gamma $|\gamma|$ ), Kolmogorov complexity $(\mathrm{K})$, permutation entropy in the theta-alpha band $(\mathrm{PE} \theta)$ and weighted symbolic mutual information in the theta-alpha band (wSMI $\theta$ ) over the 224 scalp electrodes according to the behavioral response to $\mathrm{tDCS}$. After minus before differences are presented for both non-responders $(\mathrm{R}-$ ) and responders $(\mathrm{R}+$ ) (left columns), followed by the univariate contrast between the two (middle column) and the corresponding statistical comparison using a two-steps spatial cluster-based permutation approach (right columns). Significant centro-parietal clusters were found for $|\theta|$ and $|\alpha|$ power $(p=0.0343$ and $p=0.0425)$ and for wSMI $\theta(p=0.0114)$. Absolute t-values are plotted with a red color scale when a significant cluster surviving multiple comparisons correction through a cluster-based permutation approach was found and in grey otherwise. Electrodes forming the cluster are highlighted by white circles. 


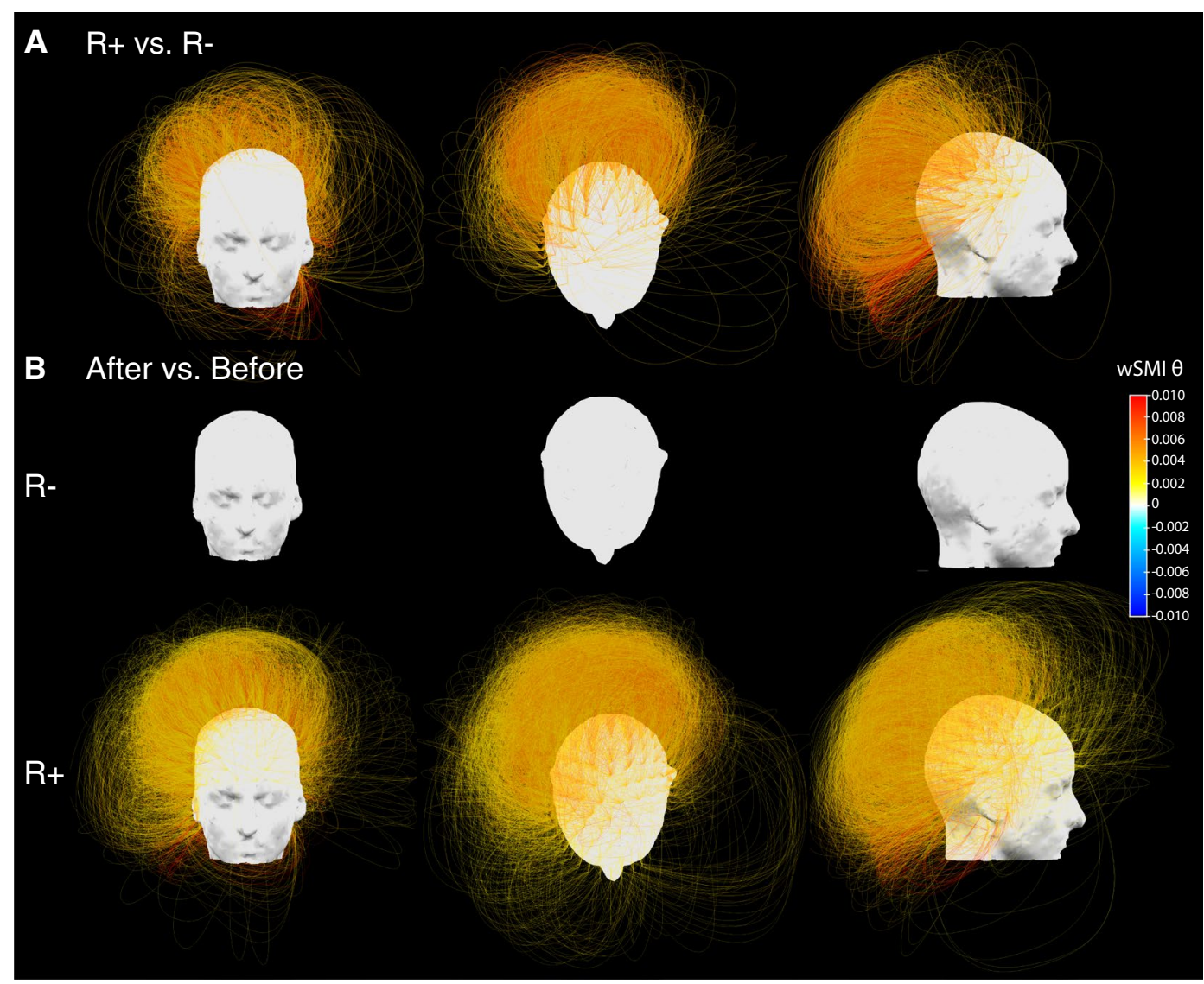

Figure 3. Functional connectivity in the theta-alpha band. (A) Three-dimensional representation of functional connectivity pre/post tDCS changes in the theta-alpha band assessed by the weighted symbolic mutual information (wSMI $\theta$ ) showing a significant increase in the centro-posterior regions in responders $(\mathrm{R}+$ ) compared to non-responders $(\mathrm{R}-)$ using cluster-based permutation analysis. Four significant clusters, involving respectively $902(p=0.01), 438(p=0.02), 363(p=0.03)$ and $245(p=0.04)$ pairs of electrodes, were identified within the same centro-posterior region. For visual clarity, the four clusters are plotted together (total of 1948 pairs). (B) Restricted pre/post contrast revealed a significant increase in the wSMI $\theta$ over centro-parietal regions after $\mathrm{tDCS}$ in $\mathrm{R}+$ (one single cluster of 5918 pairs of electrodes, $p=0.02$, see $\mathrm{B}$, bottom row), whereas no change could be detected in $\mathrm{R}-$ (see $\mathrm{B}$, upper row). Only the results surviving multiple comparisons correction through a cluster-based permutation approach are reported, consequently, only pairs of electrodes belonging to significant clusters are plotted.

from their spatial distribution. This is particularly helpful in studies involving brain-injured patients in which the identification of classical ERP components can fail due to a substantial between-subjects spatial variability. A significant difference was observed as an increase of decoding performances in $\mathrm{R}+$ versus $\mathrm{R}-$ patients (two significant clusters with $p=0.002$ and $p=0.04$ respectively, $r=0.59$ [0.07-0.79]; Fig. 4C). While $\mathrm{R}-$ patients did not show any significant cluster when comparing before and after tDCS recordings, $\mathrm{R}+$ patients did show a significant increase of trial class decoding, corresponding to two late and sustained clusters ( $200-600 \mathrm{~ms}$ after fifth sound onset; both $p<0.04 ; r=0.78$ [0.61-0.85]; Fig. 4D and SI section B6, Fig. S6B). Notably, this increase of decoding performances after tDCS assumes a square shape from around $300 \mathrm{~ms}$ to $600 \mathrm{~ms}$ on the generalization matrix, suggesting an underlying metastable brain activity in this time window ${ }^{54,55}$, evocative of the late P3b signature of conscious access to the violation of auditory novelty ${ }^{56}$.

Taken together our results show that behavioral response to tDCS was characterized by an all-or-none difference: while the $\mathrm{R}+$ group showed a significant effect including a late $\mathrm{P} 3$ signature of conscious access to violations of auditory regularities, no such response could be detected in the $\mathrm{R}$ - group, neither with a univariate nor with a multivariate approach.

Multivariate prediction of conscious state increases in responders to tDCS. Beyond univariate measures, we also assessed if a behavioral response to tDCS was associated with improvement of a multivariate EEG-based prediction of conscious state. To that end, we used the support vector machine classifier previously reported to distinguish VS/UWS from MCS using 68 resting-state EEG features (averages and fluctuations over time and space of the univariate markers described above). This algorithm was trained on a previously published database of 142 EEG recordings obtained from 98 patients (75 VS/UWS and $67 \mathrm{MCS})^{13}$. For each patient we computed the prediction of being classified MCS, before and after $\mathrm{tDCS}$, and used a non-parametric analysis of repeated measures 

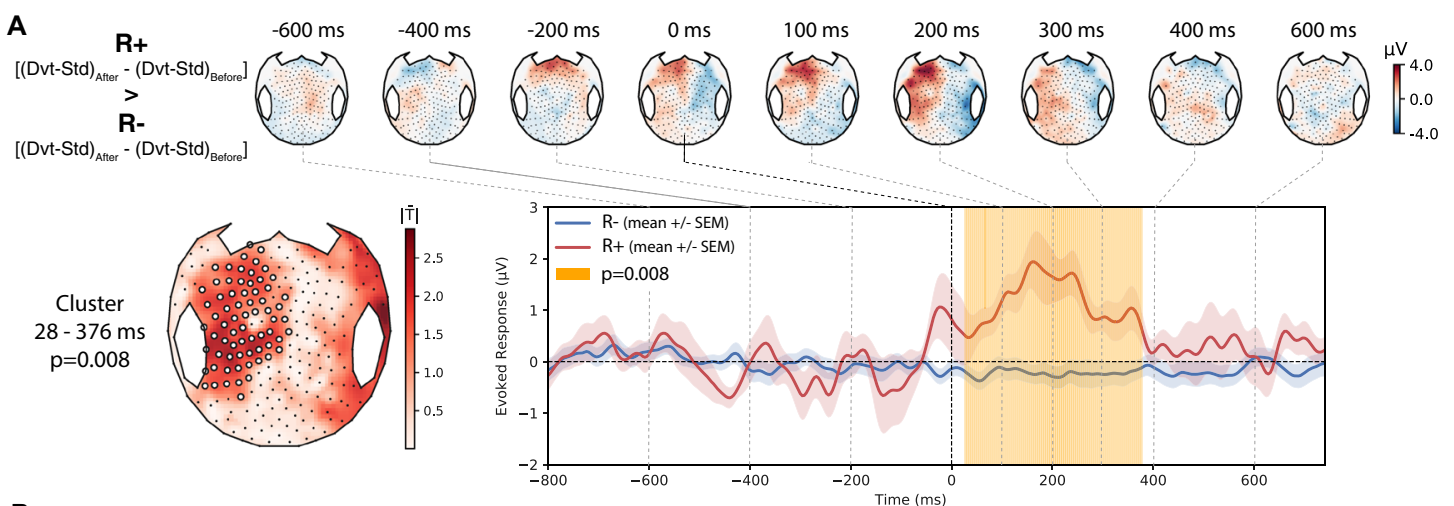

B
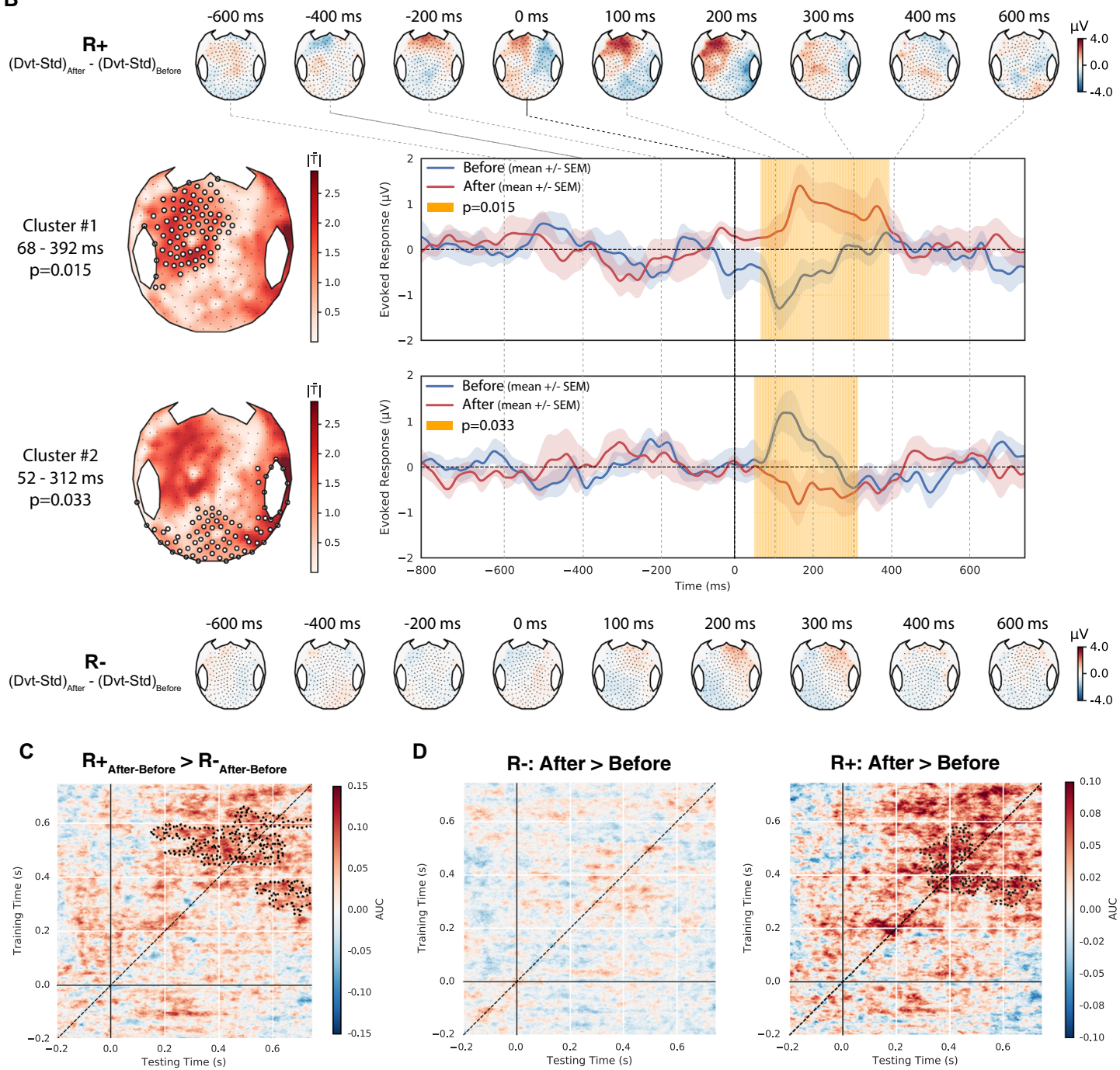

D

R-: After > Before
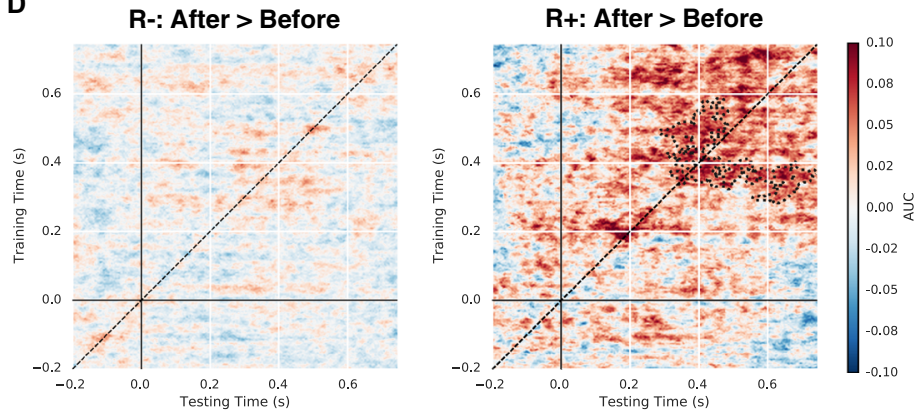

Figure 4. Neural signatures of conscious access to auditory stimuli increase after tDCS in responders. (A) Dynamics of event-related potentials elicited by tDCS in an auditory oddball paradigm (After $>$ Before difference of the Deviant (Dvt) $>$ Standard (Std) contrast), in non-responders ( - , top) and responders ( $\mathrm{R}+$, bottom) respectively. (B) A significant spatio-temporal cluster was observed over left fronto-temporal electrodes (white circles) between $28 \mathrm{~ms}$ to $376 \mathrm{~ms}$ ( $p=0.008$; left panel), and the time-course of its voltage amplitude is shown in $\mathrm{R}-$ (blue) and $\mathrm{R}+$ (red). (C) Temporal generalization decoding analysis revealed a significant increase of the decoding performances (after minus before mean AUC) in response to tDCS in R+ as compared to $\mathrm{R}-$, with two significant clusters approximately maximal around $300 \mathrm{~ms}$ and $600 \mathrm{~ms}$ respectively $(p=0.002$ and $p=0.04)$. (D) Restricted comparisons showed that while a significant increase in decoding could be observed in $\mathrm{R}+$ (significant clusters around 300 and $600 \mathrm{~ms}, p=0.03$ and $p=0.04$ ), no such effect could be found in $\mathrm{R}-$. The metastable square pattern in this late time-window is suggestive of an increased $\mathrm{P} 3 \mathrm{~b}$ component induced by tDCS in $\mathrm{R}+$. Only the results surviving multiple comparisons correction through a cluster-based permutation approach are reported. 


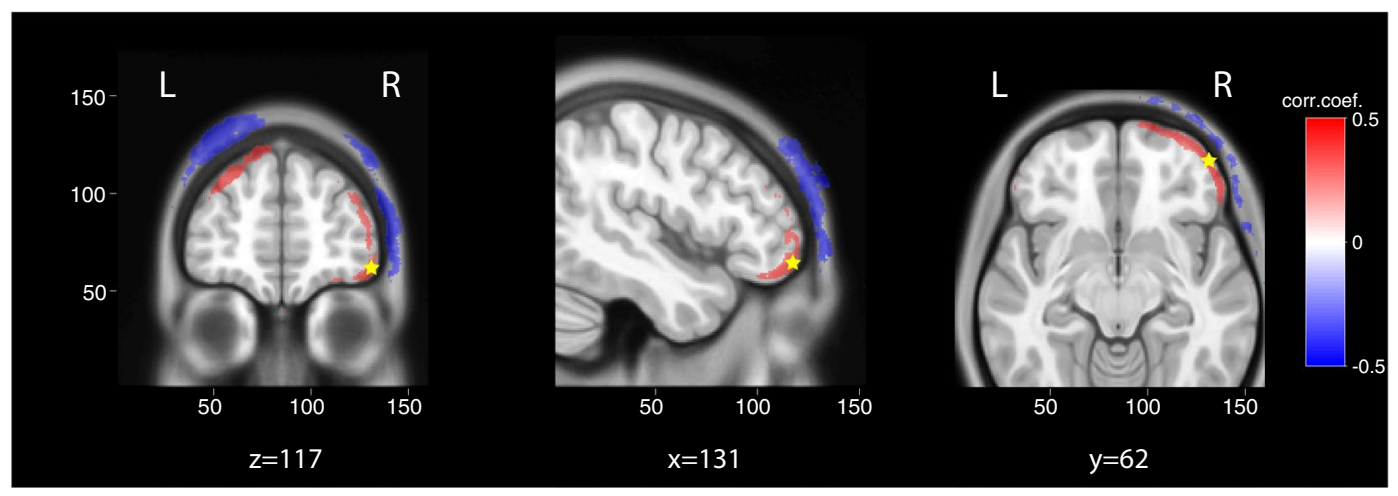

Figure 5. Correlation of electrophysiological response with electric fields magnitude. To determine the mechanism of action of $\mathrm{tDCS}$, the pre/post change in EEG multivariate prediction of consciousness was correlated with tDCS-induced electric field distribution modeled on single-subject anatomy using T1-weighted MRI ( $n=47$ patients). Correlations were restricted to areas with electric fields likely to have a physiological effect using a cut-off of mean electric fields $>0.5 \mathrm{~V} / \mathrm{m}$. Multivariate EEG prediction of consciousness significantly correlated with higher electric fields in superficial cortical areas close to the stimulating electrodes (left dorsolateral prefrontal cortex and right supraorbital cortex) and with lower electric fields in the adjacent skin (voxel-wise significant positive (red) and negative (blue) correlations with $\mathrm{p}<0.01$ uncorrected). The average correlation coefficients were 0.433 and -0.432 in the areas with positive and negative correlations respectively. A statistical analysis on the strength of these mean correlations using 10000 permutations to control for multiple comparisons yielded $\mathrm{p}=0.0069$ and $\mathrm{p}=0.0359$ respectively (similar results were obtained when analysis was limited to areas with mean electric fields $>1 \mathrm{~V} / \mathrm{m}$, not shown).

factorial design with MCS prediction as the dependent variable, the behavioral response as between-subjects factor $(\mathrm{R}+$ vs. $\mathrm{R}-)$, and stimulation as within-subjects factor (pre- vs. post-tDCS). While no main effect of either stimulation or behavioral response was observed, a significant stimulation by behavioral response interaction was present $(\mathrm{F}(1,58)=4.2, \mathrm{p}=0.045)$. Post-hoc testing with Wilcoxon signed-rank test showed that while a significant increase of MCS prediction after tDCS was present in $\mathrm{R}+$ patients (median difference of 5.0\% [0.9; 13.7], $\mathrm{p}=0.01, \mathrm{r}=0.51$ [0.19-0.63]), no effect was found in $\mathrm{R}$ - patients (median difference of $2.5 \%[-5.3 ; 7.6], \mathrm{p}=0.32, \mathrm{r}=0.10[0.0-0.29]$ ).

Electrophysiological response correlates with electric fields intensity in prefrontal cortices. So far, we showed that behavioral improvement of consciousness after tDCS was paralleled by an enrichment of objective EEG measures of both conscious state and conscious access. In order to assess whether this enrichment of EEG activity was specifically mediated by tDCS, - and if so, by which mechanisms -, we then investigated the relation between EEG changes and tDCS-induced electric fields.

Several mechanisms of action of tDCS have been proposed, including modulation of neuronal excitability ${ }^{57}$ and effects on synaptic plasticity ${ }^{58}$. These two mechanisms, which have been conclusively demonstrated in in-vitro and in-vivo animal studies are driven by the polarization of the neuronal membrane that is brought about by the applied electric field. This polarization increases linearly with field strength ${ }^{57}$ and it is therefore reasonable to assume that effects on neural function scale with electric field magnitude. There are some concerns that conventional protocols are sufficient in terms of field intensity to affect neuronal circuits ${ }^{35}$, and more recently there also have been suggestions that the effects of transcranial stimulation may result from the activation of peripheral nerves and in particular from scalp sensations which have been well-documented ${ }^{59}$. These peripheral effects also scale with electric field magnitude by virtue of the same membrane polarization of peripheral nerves. To determine the possible mechanisms of action of the stimulation in our population, and disentangle cortical vs. peripheral effects, we analyzed the relation between electric fields in brain and scalp on the one hand and EEG effects on the other.

To that end, we estimated tDCS-induced electric fields in the entire head of patients, based on available T1-weighted MRI $(n=47)$. We then correlated electric fields across patients with the pre/post difference in EEG effects. For EEG we used the multivariate predictor of conscious state described in the previous section. Correlation analysis was limited to areas with mean electric fields likely to have a physiological effect, i.e. $>0.5$ $\mathrm{V} / \mathrm{m}^{36,60}$. Areas with positive correlations surviving multiple comparisons included both left-dorsolateral and supraorbital prefrontal cortices close to stimulating electrodes $(r=0.433, p=0.007$, permutation test, see Statistical analysis in Methods section), while negative correlations were exclusively confined to the adjacent scalp $(r=-0.432, p=0.036$, permutation test, Fig. 5). It is hard to reconcile a peripheral effect of skin sensation with negative correlations, whereby stronger EEG effects would coincide with weaker sensations. Instead, the results suggest that stronger fields in frontal cortical areas improved EEG markers of consciousness.

\section{Discussion}

In this study, we characterized the behavioral and electrophysiological responses to a single session of left-dorsolateral prefrontal cortex tDCS in the largest cohort of DoC patients to date. We first showed that behavioral improvement of consciousness was associated with an increase of resting-state putative markers of conscious state, together with the emergence of a neural signature of conscious access to auditory novelty. Then, we 
linked these electrophysiological changes to tDCS by showing a positive correlation between electrophysiological response and electric fields in prefrontal cortices, while a negative correlation was observed at the scalp.

Although it is impossible to ascertain due to the open-label design, several arguments strongly support a clinically relevant and genuine impact of tDCS. First, we found no significant difference with the response rate of a previous randomized controlled trial using the same stimulation parameters ${ }^{22}(12 / 60(20 \%)$ vs. 15/55 (27\%), $\chi 2(1)=0.4885, \mathrm{p}=0.4846)$. Second, this response rate exceeded the changes associated with spontaneous fluctuations observed in the literature ${ }^{61}$. Third, no decrease of CRS-R score was observed in any of the non-responders, which again goes again spontaneous fluctuations, as these fluctuations would be expected in both directions. This is probably due to the short delay between evaluations ( $<2$ hours) that limited potential confounding factors that could account for the observed effects independently of tDCS (e.g. medications, sepsis...). In the same vein, we observed no decrease of arousal after tDCS (reflected by the CRS-R arousal subscale score). As fluctuations of behavior in these patients are thought to mainly reflect temporal fluctuations of arousal ${ }^{62}$, this goes against the presence of fatigue in our population during this short interval. Furthermore, each CRS-R scoring began with an awakening protocol, which is supposed to maximize arousal level of patients. Lastly and most importantly, both the association of the behavioral response with an enhancement of EEG signatures of consciousness and the correlation of prefrontal cortices electric fields with this objective electrophysiological response are suggestive of a direct causal effect of $\mathrm{tDCS}$ on brain function, while the negative correlation at the scalp argues against indirect effects mediated by skin sensations. The use of such objective and quantified measures of brain activity is mandatory in transcranial electric stimulation studies, not only because it allows to investigate the causal link between behavior and cognitive function and the mechanisms through which tDCS acts, but also because proper blinding with a standard sham stimulation procedure seems difficult or even impossible to achieve ${ }^{63}$.

The parallel between behavioral improvement and enhancement of putative EEG signatures of consciousness adds to our understanding of the neurophysiology of consciousness. Indeed, these results support the direct, if not causal, relationship between improvement of consciousness and increase in theta-alpha frequency power and functional connectivity (in coherence with the case reports of 4 patients ${ }^{64}$ and vagus nerve stimulation in one patient ${ }^{18}$ ). So far, evidence was merely correlational, stemming from observational studies with inter-subject comparisons of separate populations of VS/UWS and MCS patients. Importantly, the findings from the present interventional study with intra-subject design and short inter-assessment delay indicate that these markers are not only specific to consciousness, but also sensitive to subtle behavioral improvements. Our study also emphasizes the central role of posterior associative cortices and posterior long-range functional connectivity in the theta band as key elements of consciousness ${ }^{11}$, which could be the substrate for distant neural networks to generate coherent and sustained patterns of large-scale activity as proposed by the Global Workspace theory of consciousness ${ }^{65,66}$. Similarly, the all-or-none difference between $\mathrm{R}+$ and $\mathrm{R}$ - during the oddball auditory paradigm supports the postulate identifying the P300 to a specific signature of conscious access ${ }^{43,46,48,67}$. This is also consistent with previous findings of an increased P300 amplitude in MCS patients after repetitive tDCS ${ }^{24}$.

Our study also enriches the understanding of the mechanisms by which tDCS can elicit improvement of consciousness. Previous studies using fMRI in healthy subjects showed that anodal-tDCS over the left-dorsolateral prefrontal cortex could modulate the functional connectivity between prefrontal cortices and thalamus ${ }^{68}$. Yet, very few studies have investigated the electrophysiological effects of tDCS in DoC patients. Some studies, focusing on differentiating tDCS impact based on the patients' state of consciousness, showed an effect of tDCS on cortical excitability ${ }^{28}$ and long-range coherence ${ }^{29,30}$ in MCS. Our study is the first to link electrophysiological changes with behavioral response to tDCS and with electric fields magnitudes in the brain. tDCS effects on the brain are thought to rely on a direct modulation of neuronal excitability ${ }^{57}$ or of synaptic plasticity ${ }^{58}$ driven by the applied electric fields. However, some authors also proposed an indirect effect through the activation of peripheral nerves and in particular skin sensations ${ }^{59}$. The association between electric fields magnitude with an objective EEG measure, unrelated to behavioral assessment, is a strong argument for the effect of tDCS in our population. Furthermore, the significant positive correlations we found between the electrophysiological response and electric fields over frontal cortical areas and negative correlations at the skin point to a cortical origin of the tDCS-related gains and against peripheral effect of skin sensations. The left-lateralized anterior topography of the P300 component, encompassing the anodal site of tDCS, also supports a relation between stimulation sites and enhancement of specific cortical networks. Finally, some $\mathrm{R}+$ patients only improved their vigilance sub-score of the CRS-R, suggesting a cortically-mediated activation of the ascending reticular activation system. Together with the electric field distribution, the identification of specific spectral power and connectivity signatures of consciousness could pave the way for the development of more efficient stimulation tDCS strategies such as individually tailored electrode montages based on patients neuroanatomy. Indeed, the substantial heterogeneity in behavioral response to electrical stimulation ${ }^{69}$ may originate from inter-subject variability in brain anatomy underlying stimulating sites ${ }^{70}$. A recent study of prefrontal tDCS delivered during a decision-making task demonstrated that prefrontal cortical morphology differences between healthy subjects accounted for more than one third of the variability in tDCS efficacy ${ }^{71}$. This may be even more critical in DoC patients who suffer from various and severe brain lesions ${ }^{72-74}$, and in whom response to tDCS depends on residual brain metabolism and grey matter integrity ${ }^{75}$. Our innovative modeling approach could herald the individual adaptation of transcranial electrical stimulation parameters to improve its therapeutic power on disorders of consciousness.

Our study is encouraging as it provides additional evidence in favor of an effect of tDCS in DoC patient together with a mechanistic account of the improvement of consciousness by modulating residual cortical activity and cortico-cortical connectivity in this population.

\section{Methods}

In this prospective case-control study, our main goal was to evaluate the impact of tDCS on brain activity (EEG) according to the presence/absence of behavioral response. In order to do that, we did a combined behavioral and electrophysiological assessment immediately before and after a single tDCS session using the following design: 
(i) baseline behavioral assessment, (ii) baseline electrophysiological assessment, (iii) 20 min tDCS session, (iv) post-stimulation electrophysiological assessment, and (v) post-stimulation behavioral assessment (Fig. 1A, see below). Details regarding the stimulation parameters and the procedures used for behavioral and electrophysiological assessments are presented below, but it should be noted here that the whole post-stimulation evaluation was completed within approximately 45 minutes to one hour. Given that the effects on cortical excitability of a single session of tDCS are known to last at least an hour ${ }^{76-78}$, and that more prolonged behavioral effects are reported ${ }^{79,80}$, our design allowed us to capture both electrophysiological and behavioral effects of the stimulation if any.

Ethical statement. As patients were non-communicating patients, informed consents were obtained from the patients' relatives. The protocol conformed to the Declaration of Helsinki, to the French regulations, and was approved by the local ethic committee (Comité de Protection des Personnes Ile-de-France 1 (Paris, France), CPP $n^{\circ}$ 2013-A01385-40), under the code 'Recherche en soins courants'. This study was registered retrospectively on July 292019 on ClinicalTrial.gov: NCT04035655.

Population. All patients referred to the Neurology Intensive Care Unit of the Pitié-Salpêtrière university hospital (Paris, France) for an evaluation of consciousness were screened for participation in the study. This standardized evaluation includes detailed and repeated behavioral assessments, structural and functional brain-imaging recordings (standard and quantitative EEG, cognitive evoked potentials, MRI). Patients aged between 18 and 80 years with a disorder of consciousness as assessed by the Coma Recovery Scale-revised (VS, MCS or exit-MCS), stable clinical examination (even in the intensive care unit) and structural brain injury confirmed by cerebral imaging (MRI or TDM) were included in the study. Exclusion criteria were known preexisting severe neurodegenerative disease (i.e.: Alzheimer disease, Lewy body dementia,...), tDCS and/or MRI contraindications (pace-maker, metal in the head, uncovered craniectomy, refractory epilepsy), pregnant, parturient or breastfeeding women and mechanical ventilation.

Transcranial direct current stimulation (tDCS). The stimulation consisted of a single open-label 20 minutes session of anodal tDCS stimulation over the left dorsolateral prefrontal cortex of $2 \mathrm{~mA}$. The anode was placed over the left dorsolateral prefrontal cortex and the return electrode was placed over the right supraorbital frontal cortex (respectively F3 and Fp2 in the 10-20 international system EEG placement). Stimulation was delivered using Neuroelectrics Starstim 8 system ${ }^{\circledR}$, through $25 \mathrm{~cm}^{2}$ circular sponge electrodes soaked with saline solution. Impedances were kept below $10 \mathrm{k} \Omega$ during the whole stimulation session. We chose this montage to match previous studies of tDCS in DoC patients.

Behavioral evaluation. State of consciousness was assessed using the clinical gold-standard Coma Recovery Scale-Revised scale (CRS-R) which evaluates the presence or absence of responses on a set of hierarchically ordered items testing auditory, visual, motor, oromotor, communication and arousal functions. CRS-R is both quantitative (scores range from 0 to 23) and qualitative with some key behaviors defining different states of consciousness (coma, VS/UWS, MCS or exit-MCS). Response to tDCS (R+) was defined a priori by an increase in CRS-R score after stimulation compared to the CRS-R score before stimulation by contrast with no change or a decrease in CRS-R score ( $\mathrm{R}-$ ). All CRS-R were performed by trained physicians (FF, BH and PP) at the same time of the day (end of the morning). Each patient was evaluated by the same physician, and physicians were not blinded to the intervention. However, it's important to note that we used analyses based on objective EEG measures with the post-stimulation recording preceding the post-stimulation behavioral assessment, and that since all patients received the same active stimulation, both the findings of the $\mathrm{R}+\mathrm{vs}$. $\mathrm{R}-$ comparisons and the EEG-electric fields correlation are unlikely to be explained by an expectation bias.

Electroencephalography analyses. Acquisition. The electrophysiological effects of tDCS were assessed using a 5 minutes resting-state EEG and an auditory oddball ERP paradigm, derived from the previously published local-global paradigm ${ }^{48}$ designed to elicit automatic (mismatch negativity ${ }^{51,52}$ and P3a) and conscious (P3b) signatures of the detection of an auditory novelty ${ }^{49,50}$ (SI Appendix A1). Scalp EEG were recorded at a sampling rate of $250 \mathrm{~Hz}$ using a NetAmps 300 Amplifier (Electrical Geodesics, Eugene, Oregon) with a high-density sponge-based 256 channels HydroCel Geodesic Sensor Net (Electrical Geodesics) referenced to the vertex. Importantly, the EEG cap was left in place throughout the tDCS session (stimulation electrodes were slithered underneath the EEG net), and before each recording, impedances were set below $100 \mathrm{k} \Omega$.

Preprocessing. EEG data were processed using an automatized and hierarchical pipeline for artefact removal and extraction of EEG measures previously described ${ }^{13,15,47}$. Written in Python, C, and bash shell scripts and based on open-source technologies, including the software $\mathrm{MNE}^{81}$, the preprocessing workflow proceeded as follows:

EEG recordings were band-pass filtered (using a Butterworth 6th order high-pass filter at $0.5 \mathrm{~Hz}$ and a Butterworth 8th order low-pass filter at $45 \mathrm{~Hz}$ ) with $50 \mathrm{~Hz}$ and $100 \mathrm{~Hz}$ notch filters. EEG during task were cut into epochs according to the onset of the fifth sound ( $800 \mathrm{~ms}$ before and $740 \mathrm{~ms}$ after) and resting-state EEG into $800 \mathrm{~ms}$ epochs with a 550 to $850 \mathrm{~ms}$ random jitter in-between (since this step is random, the preprocessing was repeated 100 times and subsequent measures were averaged over the 100 iterations). Channels that exceeded a $150 \mu \mathrm{V}$ peak-to-peak amplitude in more than $50 \%$ of the epochs were rejected. Channels that exceeded a $\mathrm{z}$-score of 4 across all the channels mean variance were rejected. This step was repeated two times. Epochs that exceeded a $150 \mu \mathrm{v}$ peak-to-peak amplitude in more than $10 \%$ of the channels were rejected. Channels that exceeded a $\mathrm{z}$-score of 4 across all the channels mean variance (filtered with a high-pass of $25 \mathrm{~Hz}$ ) were rejected. This step was repeated two times. The remaining epochs were digitally transformed to an average reference. Rejected channels were interpolated. EEG were deemed to pass this preprocessing step if at least $75 \%$ of the channels and at least $30 \%$ 
of the epochs were kept. To allow for the assessment of the electrophysiological effects of tDCS, only sessions in which both before and after stimulation recordings (either resting-state EEG or EEG during the task) passed the preprocessing stage were included in the analysis. For all subsequent EEG analyses, we only kept scalp electrodes (224 channels over 256).

Resting-state EEG analyses. Quantitative markers: Seventeen quantitative markers in three different domains were derived from the resting-state EEG recordings as in Sitt et al. ${ }^{13}$ and Engemann et al. ${ }^{15}$ (SI Appendix A2, Table S1):

- Spectral domain:

Power spectrum density in each frequency band $(\delta: 1-4 \mathrm{~Hz} ; \theta$ : 4-8Hz; $\alpha: 8-12 \mathrm{~Hz} ; \beta: 12-30 \mathrm{~Hz} ; \gamma: 30-$ $45 \mathrm{~Hz}$ ) were computed using Fast Fourier Transformation with the Welch method with a periodogram of $512 \mathrm{~ms}$ with $400 \mathrm{~ms}$ overlap. Raw and normalized spectral power (the sum of power in a frequency band reported to the power on all frequency bands of the spectrum sum) are reported for each frequency band. Spectral entropy (characterizing the complexity of the spectrum), median spectral frequency, spectral edge 90th and 95th were computed. Both spectral power and spectral summaries were previously associated with conscious state of DoC patients ${ }^{16,82-85}$.

- Connectivity:

Functional connectivity was assessed using the weighted symbolic mutual information (wSMI). This metric, able to capture non-linear coupling between pairs of electrodes, was introduced by King et al. ${ }^{11}$. It reflects the statistical dependence of the transformation of the EEG signal into patterns of $\mathrm{k}$ discrete symbols (here $\mathrm{k}=3$ ) sampled at a time interval $(\tau)$ which determines the frequency range specificity and thus quantifies the amount of shared information between electrodes in each frequency band. In this study, we focused on the wSMI in the theta-alpha range $(4-10 \mathrm{~Hz}, \tau=32 \mathrm{~ms})$, that we called wSMI $\theta$, which was shown to have the best discriminative power across different state of consciousness (from VS/UWS to conscious subjects).

- Complexity:

We used two quantitative metrics to quantify the EEG complexity. First the Kolmogorov-Chaitin complexity, which captures algorithmic complexity of each single EEG channel by assessing the redundancy of the EEG signal. It is computed as the compressive size of the EEG signal using a variant of Lempel-Ziv algorithm and was previously used to index conscious state in human subjects from the evoked response to a TMS pulse ${ }^{86}$. Second, we measured permutation entropy in the theta band, which was successfully applied to the EEG-based detection of loss of consciousness under anesthesia ${ }^{87,88}$. As for the wSMI, this measure first relies on the transformation of the EEG into discrete symbols, before computing the entropy to quantify dynamical changes in the time series of symbols.

All the markers were first computed at the single subject level: a value was obtained for each epoch at each channel (or channels pairs for the wSMI). Values were then averaged over the epochs using the trimmed mean $80 \%$ (mean of the distribution after trimming the $10 \%$ lowest and $10 \%$ highest values, a robust estimator of central tendency ${ }^{89}$ to obtain a two-dimensional topographical representation over the 224 scalp electrodes for each subject. As the wSMI quantifies the shared information between electrodes, it was computed at each pairs of scalp electrodes $(224 \times(224-1) / 2=24976)$ and represented in a three-dimensional space. In addition, a two-dimensional representation was also obtained by resuming the value at each electrode by the median value of wSMI between one electrode and all the others. This averaging is closely related to the degree measure of a network in graph theory and highlights the sensors that have the strongest connections with other sensors, thus identifying hubs of connections. All analyses were done at the group level, using the mean across groups.

Multivariate Pattern Analysis (MVPA): In addition to the effect on single markers, we assessed the overall effect of tDCS on brain activity using a MVPA approach as previously described by Sitt et al. ${ }^{13}$. We used a linear support vector classifier to predict the MCS diagnosis (as opposed to VS/UWS) from the resting-state EEG derived markers. To that end, the 17 previously described markers were summarized using all four combinations between their averages and fluctuations over time (trimmed mean $80 \%$ and standard deviation over the epochs) and space (mean and standard deviation over the scalp channels): trimmed mean over the epochs and mean over the scalp, trimmed mean over the epochs and standard deviation over the scalp, standard deviation over the epochs and mean over the scalp and finally, standard deviation over the epochs and standard deviation over the scalp. This resulted in $68(=17 \times 4)$ features for each subject (SI Appendix B2, Table S1). The support vector classifier algorithm using these features was trained against a previously published database of 142 EEG recordings (68 MCS and $75 \mathrm{VS} / \mathrm{UWS}$ ) in 98 different patients ${ }^{13,15}$ with a $20 \%$ feature selection (keeping only the $20 \%$ markers showing the highest discrimination performance between VS and MCS patients on univariate F-tests in the training dataset, see SI Appendix B2, Table S1) and 5-fold stratified cross-validation with a penalization parameter $C$, chosen by nested cross-validation among the values $=\left[10^{-6} 10^{-5} 10^{-4} 10^{-5} 10^{-4} 10^{-3} 10^{-2} 10^{-1}\right]$ using a grid-search method. The algorithm was then tested on each participants' resting-state EEG to compute the classifier's prediction of being MCS according to the brain activity both before and after the tDCS session separately. We used the Platt scaling method to obtain probabilities (between 0 and 1 ) from the classifier's output. The change induced by tDCS was obtained by subtracting the probability when tested on the resting-state EEG before stimulation from the probability when tested on the resting-state EEG after stimulation. We used the scikit-learn software for machine learning ${ }^{90}$.

Auditory oddball analyses. For the analysis of EEG during the auditory oddball paradigm, data were further low-pass filtered at $20 \mathrm{~Hz}$ and baseline corrected over the first $800 \mathrm{~ms}$ (from the beginning of the trial to the onset of the fifth sound). 
ERP topographies: We first analyzed group-level event-related potentials (ERP) elicited by the auditory oddball paradigm. These were obtained by averaging trials of each condition with the trimmed mean $80 \%$ (across trial) over the scalp channels at the subject-level. We then computed group-level ERP to deviant minus standard trials, before and after tDCS, and compared R+ with R- (or VS/UWS with MCS \& exit-MCS) with the following contrast: $\left[[\text { Deviant }- \text { Standard }]_{\text {after }}-[\text { Deviant }- \text { Standard }]_{\text {before }}\right] \mathrm{R}+$ vs. $\left[[\text { Deviant }- \text { Standard }]_{\text {after }}-[\right.$ Deviant - St andard $\left.]_{\text {before }}\right] \mathrm{R}-$. Results are reported by the mean \pm standard error of the mean.

Temporal generalization decoding: As classical ERP analyses are prone to a high inter-subject spatial variability, especially in brain-lesioned patients, we completed the topographical analysis with the analysis of the temporal dynamic of the ERP. To that end, with used the Temporal Generalization decoding method described by King et al. ${ }^{53}$. This MVPA approach relies on training a classifier at each time point of the trial to distinguish deviant from standard trials at the single-subject level. Each classifier was then tested not only on the time sample it was trained on, but also on every other samples of the trial to see if decoding performances generalized in time. This procedure thus allows to extract patterns of brain activation associated with different cognitive tasks based on their temporal dynamics. This procedure has previously been applied to an auditory oddball paradigm and showed that the violation of auditory regularity led to two kinds of neural activation patterns: an automatic, early and short-lived response to auditory novelty and a late and sustained $(200-700 \mathrm{~ms})$ pattern associated with the conscious access to the auditory novelty. This latter pattern probably reflects the same process as the one engaged in the generation of the P300 during the same paradigm ${ }^{56}$. Here we applied this MVPA procedure using linear support vector classifier (with 10 iterations of a stratified 5-fold cross-validation). Performances of the classifiers were expressed as the area under the curve (AUC).

Correlation of electric fields with EEG analysis. In order to estimate field distributions in individual patients we follow standard practice, namely, first we segment the head volume into different tissue compartments, then place virtual electrodes, and then use finite-element modeling to estimate current flow in the entire volume. We then correlate these field magnitudes with the EEG measure and use permutation statistic to determine statistical significance on the strength of these correlations. These steps are described in detail in the following sections.

MRI acquisition. T1-weighted MRI images were acquired during the patient hospitalization in 56 out of the 60 patients of the study, on a $3 \mathrm{~T}$ General Electric Signa system (Milwaukee, WI) with a varying number of slices (from 152 to 234 ) and voxel sizes (from 0.42188 to $0.4883 \mathrm{~mm}^{3}$ ). This variability was not an issue because MRIs were down-sampled to a common $1 \mathrm{~mm}^{3}$ isotropic resolution in the process of co-registering the individual brains with a common standard (MNI152 standard head, see below).

Tissue segmentation. Segmentation of the head volume is based on the T1-weighted MRI of each patient. Segmentation distinguishes the following six tissue types: scalp, skull, cerebrospinal fluid (CSF), gray matter, white matter, and air cavities (e.g. sinuses). Due to the substantial abnormality of these patients' anatomy, popular segmentation algorithms developed for normal heads (e.g., Unified Segmentation as implemented in SPM8 ${ }^{91}$ often fail to accurately classify different tissues, especially the enlarged ventricles in the brain of these patients). Therefore, we adopted a machine learning approach. Specifically, a volumetric deep convolutional neural network (DCNN) was trained with hand segmentations of MRI's from 37 ischemic stroke heads available from an unrelated project ${ }^{92}$. The trained DCNN was used to segment the 56 MRIs. Since the DCNN requires the input data to have the same size, the MRIs were first registered and resampled to a common space with $1 \mathrm{~mm}^{3}$ isotropic resolution before entering the DCNN. This was done by using the co-register function in SPM $8^{93}$. Post-processing of the segmentations was done with a dense $3 \mathrm{D}$ conditional random field (CRF) ${ }^{94}$. This step makes final corrections to the output of the neural network by incorporating known morphological constraints. These constraints are hand-coded in the weights of the CRF, which penalize neighboring tissue categories that are unrealistic, for example, brain cannot be next to air. This ensures among other things, that brain tissue is surrounded by the $\mathrm{CSF}^{95}$. For validation purposes, two MRIs of the present patient population with prominent enlarged ventricles were manually segmented. We find that improved performance in terms of Dice score for the DCNN as compared to SPM8 (SI section B7, Fig. S7). In the end, 47 MRI could be successfully segmented using this procedure and 9 had to be discarded due to insufficient quality (mainly because of movement artifacts).

Current flow modeling. The segmented tissues output from the DCNN were feed into an open-source software ROAST $^{96}$ to model the current-flow in the entire head volume. Electrodes were placed automatically by ROAST at positions F3 and Fp2 on the scalp. Electrodes were modeled as a disc with $25 \mathrm{~cm}^{2}$ cross-sectional area and $2 \mathrm{~mm}$ thickness. Finite element meshing and solving were subsequently performed in ROAST fully automatically. Boundary conditions were set as $2 \mathrm{~mA}$ current injected at electrode F3, and $2 \mathrm{~mA}$ flowing out of electrode Fp2. Literature tissue conductivity values were used ${ }^{97}$. To facilitate the voxel-level correlation analysis that is described in the next section, we registered and warped the electric field magnitude in each subject to the MNI152 standard head (version $2009 \mathrm{a}^{98,99}$ ). This was done by using the Unified Segmentation function ${ }^{91}$ together with the DARTEL toolbox in $\mathrm{SPM}^{100}$. Specifically, Unified Segmentation was run on the MRI of each subject, using the MNI152 standard head as the reference, during which a warping field from individual MRI to the standard head was estimated. The DARTEL function was then used to apply the estimated warping field on the electric field magnitude output from ROAST. In this way the field magnitude in all the subjects are co-registered with the voxel space of the standard head. The advantage of DARTEL over conventional affine transform is that it is capable of applying different warping field locally to different part of the image volume, and thus transforming different head shapes in each subject to a common shape represented by the standard head. This detailed alignment is important as fields differ drastically between cortical surface, CSF, bone and skin (due to very different conductances). Since 
these structures are all very thin, incorrect alignment results in a mismatch of tissue types across subjects, with potentially larger errors when correlating the EEG regressor variable with field magnitudes across subjects.

Statistical analyses. We first assessed the effect of prefrontal tDCS on both the behavior and a set of comprehensive quantitative electrophysiological markers of consciousness. To that end, we compared the EEG-derived markers and the ERP according to the behavioral response to tDCS. To further understand the origin of significant differences if any, we also tested the after vs. before difference in each group separately when appropriate. The same types of analyses were performed contrasting tDCS-induced effect according to the state of consciousness before stimulation (VS/UWS vs. MCS and exit-MCS). Lastly, to understand the origin of these electrophysiological changes after tDCS, we correlated electric fields modeled on individual patients' brain anatomy with the EEG multivariate prediction of consciousness.

Behavioral analysis and population characteristics. Population baseline characteristics (age, sex, time since brain injury, etiology and CRS-R scores) between R- and R+ and between VS/UWS and MCS \& exit-MCS were compared using a Mann-Whitney-U for continuous data and the $\chi 2$ test for categorical variables as appropriate. In order to assess if there were differences in EEG data preprocessing between responders and non-responders, we used a non-parametric factorial analysis of repeated measures design. This procedure consists in a standard repeated measures analysis of variance (ANOVA) on aligned and rank transformed data ${ }^{101}$ allowing for a more accurate assessment of the interaction term than with standard rank transformation. In this mixed 2-by-2 design, we tested the effects of the behavioral response (between-subjects factor: responder or non-responder) and tDCS stimulation (within-subjects factor: before and after tDCS) on the number of channels/epochs rejected (dependent variable).

Electrophysiological data. For the analysis of multivariate MCS prediction, we used the same non-parametric factorial analysis with the behavioral response as between-subjects factor and tDCS stimulation as the within-subjects factor (before and after tDCS). All others resting-state EEG and auditory paradigm EEG analyses involved comparisons at multiple sensors and/or timepoints requiring a control procedure through permutations. However, there are debates on how permutations should be done in such a factorial design. In order to obtain a simpler contrast for the interaction term, we resumed the within-subjects effect of stimulation to the after minus before subtraction: [post - pre] in $\mathrm{R}+\mathrm{vs}$. [post - pre] in $\mathrm{R}-$. This analysis allowed us to assess the interaction while robustly controlling for multiple comparisons through a cluster-based permutation approach ${ }^{102}$. This two-step robust non-parametric procedure is able to control for the multiple comparisons over sensors and time by acknowledging the dependence between spatially close electrodes and timepoints. First, a test statistic between the two groups was computed at each sample, i.e. each channel for the EEG markers, each channel-timepoint pair for the ERP and each channels pair for the temporal generalization decoding. Respectively, spatial, spatiotemporal and spatio-spatial clusters were constructed using a connectivity matrix with the inclusion of the test statistic corresponding to a first step statistic p-value of 0.05 . For each cluster identified, the sum of test statistic over sensors included in the cluster (the cluster mass) was computed. The second step relied on the creation of surrogate datasets by 10000 random permutations of the groups' labels to construct the distribution of the cluster mass under the null hypothesis that data from both groups are drawn from the same distribution. This allowed to estimate the probability of observing randomly each cluster constructed with the genuine data. As the first step statistic, we used a Welch test (unequal variance t-test, t-statistic) for the resting-state EEG markers analyses and the ERP topographies to compare mean voltage and a Mann-Whitney-U test (z-statistic) for multivariate analyses to compare median AUCs. For the additional analyses testing differences between after and before stimulation separately in each group, we used a dependent t-test and Wilcoxon Signed-Ranked test respectively. A type I error of $5 \%$ was considered as significant. When present, we reported clusters' effect size, defined as the effect size computed after averaging over the electrodes and/or timepoints constituting the cluster in both populations. For the resting-state EEG an ERP topographies, we used the Hedges' $g$ coefficient ${ }^{103}$, which is an approximation of Cohen's d coefficient less prone to upward bias for small sample size. For the multivariate analyses, we reported the effect size measure $r: r=\frac{z}{\sqrt{N}}$. Where $z$ is the z-statistic of the Mann-Whitney-U or Wilcoxon test and $N$ the size of the population ${ }^{104}$ (SI Appendix A2). All effect sizes are reported with their bootstrapped 95\% confidence interval computed using 10000 iterations.

Correlation of electric fields with EEG multivariate prediction of consciousness. To better characterize the potential mechanisms of action of tDCS in our cohort, we correlated electric fields magnitude distribution to the pre/ post difference in multivariate prediction of consciousness. We chose this parameter for several reasons. First, the electrophysiological assessment was entirely automated and thus not susceptible to experimenter bias, in contrast to the behavioral assessment by the clinician which was not blinded. Second, the behavioral assessment with the CRS-R scale only gave a binary answer, with improvements in 9 responders. This small number of binary outcomes was not adequately powered to perform a whole-brain statistical analysis, in contrast to the graded outcomes of the electrophysiological assessment which was available for all 47 patients. Third, it is quite conceivable that there were neurophysiological changes that were not detected with the clinical behavioral assessment. Lastly, among all electrophysiological markers computed, we chose the MVPA prediction of consciousness because it systematically outperformed single EEG markers in predicting patients state of consciousness in previous studies $^{81}$. The individual subjects pre/post differences were correlated with the individuals' electric fields in each voxel with mean electric field magnitude $>0.5 \mathrm{~V} / \mathrm{m}$ using Pearson correlation coefficients. This cut-off of electric field magnitude of at least $0.5 \mathrm{~V} / \mathrm{m}$ in the average over 47 subjects was chosen based on fields magnitudes that are considered necessary to affect neural function (usually $1 \mathrm{~V} / \mathrm{m}$ or higher ${ }^{39}$, though some effects were reported with fields as low as $0.25 \mathrm{~V} / \mathrm{m}^{38}$; at a mean of $0.5 \mathrm{~V} / \mathrm{m}$ some subjects will have weaker, other subjects stronger electric 
fields in those areas). For the uncorrected statistics we used a cut-off of $\mathrm{p}=0.01$, corresponding to a correlation coefficient of 0.327 for positive correlations and -0.327 for negative correlations ( $\mathrm{N}=47$ patients). To quantify the overall strength of these correlations and the probability of observing it by chance we took the mean of the correlation coefficients in those areas and used shuffle statistics with 10,000 random permutations of patients' labels. Note that the computational estimates of electric fields vary smoothly within a given tissue, such that correlation of one voxel naturally results in correlation of many neighboring voxels. Thus, extended areas of correlation (above the $1 \%$ threshold) are expected even for the null hypothesis of no correlation. As a result, size of the areas or the integral of the correlation over the area is not an appropriate metric. This motivated us to base the shuffle statistic more directly on the mean correlation coefficient as a measure of the strength of correlation.

Softwares. All statistical analyses were performed with python using scipy $y^{105}$, mne-python $^{81}$ and scikit-learn ${ }^{90}$ packages, except for the non-parametric factorial analysis performed in R statistical software with the $A R T o o l^{106}$ package and electric fields analysis performed in Matlab software. Throughout all EEG analyses, from preprocessing to statistical analyses, we tried to comply with the CODIBAS-MEEG Best Practices in Data Analysis and Sharing in Neuroimaging using MEEG initiative ${ }^{107}$.

\section{Data availability}

The data that support the findings of this study are available from the corresponding author, upon reasonable request.

Received: 28 June 2019; Accepted: 19 February 2020;

Published online: 09 March 2020

\section{References}

1. Giacino, J. T. et al. The minimally conscious state definition and diagnostic criteria. Neurology 58, 349-353 (2002).

2. Giacino, J. T. \& Kalmar, K. Diagnostic and prognostic guidelines for the vegetative and minimally conscious states. Neuropsychol. Rehabil. 15, 166-174 (2005).

3. Naccache, L. Minimally conscious state or cortically mediated state? Brain J. Neurol. 141, 949-960 (2018).

4. Owen, A. M. et al. Detecting awareness in the vegetative state. Science 313, 1402 (2006).

5. Monti, M. M. et al. Willful modulation of brain activity in disorders of consciousness. N. Engl. J. Med. 362, 579-589 (2010).

6. Cruse, D. et al. Bedside detection of awareness in the vegetative state: a cohort study. The Lancet 378, 2088-2094 (2012).

7. Kondziella, D., Friberg, C. K., Frokjaer, V. G., Fabricius, M. \& Møller, K. Preserved consciousness in vegetative and minimal conscious states: systematic review and meta-analysis. J. Neurol. Neurosurg. Psychiatry 87, 485-492 (2016).

8. Stender, J. et al. The Minimal Energetic Requirement of Sustained Awareness after Brain Injury. Curr. Biol. 26, 1494-1499 (2016).

9. Faugeras, F. et al. Probing consciousness with event-related potentials in the vegetative state. Neurology 77, 264-268 (2011).

10. Faugeras, F. et al. Event related potentials elicited by violations of auditory regularities in patients with impaired consciousness. Neuropsychologia 50, 403-418 (2012).

11. King, J.-R. et al. Information Sharing in the Brain Indexes Consciousness in Noncommunicative Patients. Curr. Biol. 23, 1914-1919 (2013).

12. King, J. R. et al. Single-trial decoding of auditory novelty responses facilitates the detection of residual consciousness. NeuroImage 83, 726-738 (2013).

13. Sitt, J. D. et al. Large scale screening of neural signatures of consciousness in patients in a vegetative or minimally conscious state. Brain 137, 2258-2270 (2014).

14. Chennu, S. et al. Brain networks predict metabolism, diagnosis and prognosis at the bedside in disorders of consciousness. Brain J. Neurol. 140, 2120-2132 (2017).

15. Engemann, D. A. et al. Robust EEG-based cross-site and cross-protocol classification of states of consciousness. Brain J. Neurol. 141, 3179-3192 (2018).

16. Chennu, S. et al. Spectral Signatures of Reorganised Brain Networks in Disorders of Consciousness. PLoS Comput. Biol. 10, e1003887 (2014).

17. Schiff, N. D. et al. Behavioural improvements with thalamic stimulation after severe traumatic brain injury. Nature 448, 600-603 (2007).

18. Corazzol, M. et al. Restoring consciousness with vagus nerve stimulation. Curr. Biol. CB 27, R994-R996 (2017).

19. Lemaire, J.-J. et al. Deep brain stimulation in five patients with severe disorders of consciousness. Ann. Clin. Transl. Neurol. 5, 1372-1384 (2018).

20. Bourdillon, P., Hermann, B., Sitt, J. D. \& Naccache, L. Electromagnetic Brain Stimulation in Patients With Disorders of Consciousness. Front. Neurosci. 13 (2019).

21. Angelakis, E. et al. Transcranial Direct Current Stimulation Effects in Disorders of Consciousness. Arch. Phys. Med. Rehabil. 95, 283-289 (2014).

22. Thibaut, A., Bruno, M.-A., Ledoux, D., Demertzi, A. \& Laureys, S. tDCS in patients with disorders of consciousness: shamcontrolled randomized double-blind study. Neurology 82, 1112-1118 (2014).

23. Thibaut, A. et al. Controlled clinical trial of repeated prefrontal tDCS in patients with chronic minimally conscious state. Brain Inj. 1-9 https://doi.org/10.1080/02699052.2016.1274776 (2017).

24. Zhang, Y. et al. Transcranial Direct Current Stimulation in Patients with Prolonged Disorders of Consciousness: Combined Behavioral and Event-Related Potential Evidence. Front. Neurol. 8, (2017).

25. Wu, M. et al. Efficiency of Repetitive Transcranial Direct Current Stimulation of the Dorsolateral Prefrontal Cortex in Disorders of Consciousness: A Randomized Sham-Controlled Study. Neural Plast. 2019, 1-11 (2019).

26. Guo, Y. et al. Effects of Long-Lasting High-Definition Transcranial Direct Current Stimulation in Chronic Disorders of Consciousness: A Pilot Study. Front. Neurosci. 13, 412 (2019).

27. Huang, W. et al. Repeated stimulation of the posterior parietal cortex in patients in minimally conscious state: A sham-controlled randomized clinical trial. Brain Stimulat. 10, 718-720 (2017).

28. Bai, Y. et al. TDCS modulates cortical excitability in patients with disorders of consciousness. NeuroImage Clin., https://doi. org/10.1016/j.nicl.2017.01.025 (2017).

29. Bai, Y. et al. Fronto-parietal coherence response to tDCS modulation in patients with disorders of consciousness. Int. J. Neurosci. 128, 587-594 (2018).

30. Cavinato, M. et al. Behavioural and electrophysiological effects of tDCS to prefrontal cortex in patients with disorders of consciousness. Clin. Neurophysiol. Off. J. Int. Fed. Clin. Neurophysiol. 130, 231-238 (2019). 
31. Estraneo, A. et al. Repeated transcranial direct current stimulation in prolonged disorders of consciousness: A double-blind crossover study. J. Neurol. Sci. 375, 464-470 (2017).

32. Martens, G. et al. Randomized controlled trial of home-based 4-week tDCS in chronic minimally conscious state. Brain Stimulat. https://doi.org/10.1016/j.brs.2018.04.021 (2018).

33. Horvath, J. C., Forte, J. D. \& Carter, O. Quantitative Review Finds No Evidence of Cognitive Effects in Healthy Populations From Single-session Transcranial Direct Current Stimulation (tDCS). Brain Stimulat. 8, 535-550 (2015).

34. Horvath, J. C., Forte, J. D. \& Carter, O. Evidence that transcranial direct current stimulation (tDCS) generates little-to-no reliable neurophysiologic effect beyond MEP amplitude modulation in healthy human subjects: A systematic review. Neuropsychologia 66, 213-236 (2015)

35. Vöröslakos, M. et al. Direct effects of transcranial electric stimulation on brain circuits in rats and humans. Nat. Commun. 9 (2018).

36. Liu, A. et al. Immediate neurophysiological effects of transcranial electrical stimulation. Nat. Commun. 9, 5092 (2018).

37. Underwood, E. Cadaver study challenges brain stimulation methods. Science 352, 397-397 (2016).

38. Ruhnau, P., Rufener, K. S., Heinze, H.-J. \& Zaehle, T. Sailing in a sea of disbelief: In vivo measurements of transcranial electric stimulation in human subcortical structures. Brain Stimulat. 11, 241-243 (2018).

39. Opitz, A., Falchier, A., Linn, G. S., Milham, M. P. \& Schroeder, C. E. Limitations of ex vivo measurements for in vivo neuroscience. Proc. Natl. Acad. Sci. 114, 5243-5246 (2017).

40. Naro, A. et al. Unravelling motor networks in patients with chronic disorders of consciousness: A promising minimally invasive approach. Brain Res. 1646, 262-268 (2016).

41. Lefaucheur, J.-P. et al. Evidence-based guidelines on the therapeutic use of transcranial direct current stimulation (tDCS). Clin. Neurophysiol. 128, 56-92 (2017).

42. Dehaene, S. \& Naccache, L. Towards a cognitive neuroscience of consciousness: basic evidence and a workspace framework. Cognition 79, 1-37 (2001).

43. Sergent, C., Baillet, S. \& Dehaene, S. Timing of the brain events underlying access to consciousness during the attentional blink. Nat. Neurosci. 8, 1391-1400 (2005).

44. Sigman, M. \& Dehaene, S. Brain Mechanisms of Serial and Parallel Processing during Dual-Task Performance. J. Neurosci. 28, 7585-7598 (2008).

45. Melloni, L., Schwiedrzik, C. M., Muller, N., Rodriguez, E. \& Singer, W. Expectations Change the Signatures and Timing of Electrophysiological Correlates of Perceptual Awareness. J. Neurosci. 31, 1386-1396 (2011).

46. Del Cul, A., Baillet, S. \& Dehaene, S. Brain dynamics underlying the nonlinear threshold for access to consciousness. PLoS Biol 5, e260 (2007).

47. Engemann, D. et al. Automated Measurement and Prediction of Consciousness in Vegetative and Minimally Conscious Patients. in (2015).

48. Bekinschtein, T. A. et al. Neural signature of the conscious processing of auditory regularities. Proc. Natl. Acad. Sci. 106, 1672-1677 (2009).

49. Squires, N. K., Squires, K. C. \& Hillyard, S. A. Two varieties of long-latency positive waves evoked by unpredictable auditory stimuli in man. Electroencephalogr. Clin. Neurophysiol. 38, 387-401 (1975).

50. Polich, J. Updating P300: An Integrative Theory of P3a and P3b. Clin. Neurophysiol. Off. J. Int. Fed. Clin. Neurophysiol. 118, 2128-2148 (2007).

51. Näätänen, R., Gaillard, A. W. \& Mäntysalo, S. Early selective-attention effect on evoked potential reinterpreted. Acta Psychol. (Amst.) 42, 313-329 (1978).

52. Näätänen, R., Paavilainen, P., Rinne, T. \& Alho, K. The mismatch negativity (MMN) in basic research of central auditory processing: a review. Clin. Neurophysiol. Off. J. Int. Fed. Clin. Neurophysiol. 118, 2544-2590 (2007).

53. King, J.-R. \& Dehaene, S. Characterizing the dynamics of mental representations: the temporal generalization method. Trends Cogn. Sci. 18, 203-210 (2014).

54. Schurger, A., Sarigiannidis, I., Naccache, L., Sitt, J. D. \& Dehaene, S. Cortical activity is more stable when sensory stimuli are consciously perceived. Proc. Natl. Acad. Sci. USA 112, E2083-2092 (2015).

55. Charles, L., King, J.-R. \& Dehaene, S. Decoding the dynamics of action, intention, and error detection for conscious and subliminal stimuli. J. Neurosci. Off. J. Soc. Neurosci. 34, 1158-1170 (2014).

56. King, J.-R., Gramfort, A., Schurger, A., Naccache, L. \& Dehaene, S. Two distinct dynamic modes subtend the detection of unexpected sounds. PloS One 9, e85791 (2014).

57. Bikson, M. et al. Effects of uniform extracellular DC electric fields on excitability in rat hippocampal slices in vitro. J. Physiol. 557, 175-190 (2004).

58. Kronberg, G., Bridi, M., Abel, T., Bikson, M. \& Parra, L. C. Direct Current Stimulation Modulates LTP and LTD: Activity Dependence and Dendritic Effects. Brain Stimulat. 10, 51-58 (2017).

59. Asamoah, B., Khatoun, A. \& Mc Laughlin, M. tACS motor system effects can be caused by transcutaneous stimulation of peripheral nerves. Nat. Commun. 10, 266 (2019).

60. Reato, D., Rahman, A., Bikson, M. \& Parra, L. C. Low-intensity electrical stimulation affects network dynamics by modulating population rate and spike timing. J. Neurosci. Off. J. Soc. Neurosci. 30, 15067-15079 (2010).

61. Wannez, S. et al. The repetition of behavioral assessments in diagnosis of disorders of consciousness. Ann. Neurol. 81, 883-889 (2017).

62. Bekinschtein, T., Cologan, V., Dahmen, B. \& Golombek, D. You are only coming through in waves: wakefulness variability and assessment in patients with impaired consciousness. Prog. Brain Res. 177, 171-189 (2009).

63. Turi, Z. et al. Blinding is compromised for transcranial direct current stimulation at $1 \mathrm{~mA}$ for $20 \mathrm{~min}$ in young healthy adults. Eur. J. Neurosci. 0 (2019).

64. Bareham, C. A. et al. Longitudinal Bedside Assessments of Brain Networks in Disorders of Consciousness: Case Reports From the Field. Front. Neurol. 9, 676 (2018).

65. Dehaene, S., Changeux, J.-P., Naccache, L., Sackur, J. \& Sergent, C. Conscious, preconscious, and subliminal processing: a testable taxonomy. Trends Cogn. Sci. 10, 204-211 (2006).

66. Dehaene, S. \& Changeux, J.-P. Experimental and Theoretical Approaches to Conscious Processing. Neuron 70, 200-227 (2011).

67. Naccache, L., Marti, S., Sitt, J. D., Trübutschek, D. \& Berkovitch, L. Why the P3b is still a plausible correlate of conscious access? A commentary on Silverstein et al., 2015. Cortex 85, 126-128 (2016).

68. Stagg, C. J. et al. Widespread modulation of cerebral perfusion induced during and after transcranial direct current stimulation applied to the left dorsolateral prefrontal cortex. J. Neurosci. Off. J. Soc. Neurosci. 33, 11425-11431 (2013).

69. Wiethoff, S., Hamada, M. \& Rothwell, J. C. Variability in Response to Transcranial Direct Current Stimulation of the Motor Cortex. Brain Stimul. Basic Transl. Clin. Res. Neuromodulation 7, 468-475 (2014).

70. Kim, J.-H. et al. Inconsistent outcomes of transcranial direct current stimulation may originate from anatomical differences among individuals: Electric field simulation using individual MRI data. Neurosci. Lett. 564, 6-10 (2014).

71. Filmer, H. L., Ehrhardt, S. E., Shaw, T. B., Mattingley, J. B. \& Dux, P. E. The efficacy of transcranial direct current stimulation to prefrontal areas is related to underlying cortical morphology. NeuroImage 196, 41-48 (2019).

72. Fernández-Espejo, D. et al. Diffusion weighted imaging distinguishes the vegetative state from the minimally conscious state. NeuroImage 54, 103-112 (2011). 
73. Perri, C. D. et al. Neural correlates of consciousness in patients who have emerged from a minimally conscious state: a crosssectional multimodal imaging study. Lancet Neurol. 15, 830-842 (2016).

74. Annen, J. et al. Regional brain volumetry and brain function in severely brain-injured patients: Regional Brain Volumetry and Function in DOC. Ann. Neurol. 83, 842-853 (2018).

75. Thibaut, A. et al. Clinical Response to tDCS Depends on Residual Brain Metabolism and Grey Matter Integrity in Patients With Minimally Conscious State. Brain Stim 8, 1116-1123 (2015).

76. Nitsche, M. A. \& Paulus, W. Sustained excitability elevations induced by transcranial DC motor cortex stimulation in humans. Neurology 57, 1899-1901 (2001).

77. Ardolino, G., Bossi, B., Barbieri, S. \& Priori, A. Non-synaptic mechanisms underlie the after-effects of cathodal transcutaneous direct current stimulation of the human brain. J. Physiol. 568, 653-663 (2005).

78. Zappasodi, F. et al. Safety and effects on motor cortex excitability of five anodal transcranial direct current stimulation sessions in 24hours. Neurophysiol. Clin. 49, 19-25 (2019).

79. Shekhawat, G. S., Stinear, C. M. \& Searchfield, G. D. Transcranial Direct Current Stimulation Intensity and Duration Effects on Tinnitus Suppression: Neurorehabil. Neural Repair, https://doi.org/10.1177/1545968312459908 (2012).

80. Lefaucheur, J.-P. A comprehensive database of published tDCS clinical trials (2005-2016). Neurophysiol. Clin. Neurophysiol. 46, 319-398 (2016).

81. Gramfort, A. et al. MNE software for processing MEG and EEG data. NeuroImage 86, 446-460 (2014).

82. Lehembre, R. et al. Resting-state EEG study of comatose patients: a connectivity and frequency analysis to find differences between vegetative and minimally conscious states. Funct. Neurol. 27, 41-47 (2012).

83. Fingelkurts, A. A., Fingelkurts, A. A., Bagnato, S., Boccagni, C. \& Galardi, G. EEG oscillatory states as neuro-phenomenology of consciousness as revealed from patients in vegetative and minimally conscious states. Conscious. Cogn. 21, 149-169 (2012).

84. Schnakers, C. et al. Diagnostic and prognostic use of bispectral index in coma, vegetative state and related disorders. Brain Inj. 22, 926-931 (2008).

85. Gosseries, O. et al. Automated EEG entropy measurements in coma, vegetative state/unresponsive wakefulness syndrome and minimally conscious state. Funct. Neurol. 26, 25-30 (2011).

86. Casali, A. G. et al. A theoretically based index of consciousness independent of sensory processing and behavior. Sci. Transl. Med. 5, 198ra105-198ra105 (2013).

87. Jordan, D., Stockmanns, G., Kochs, E. F., Pilge, S. \& Schneider, G. Electroencephalographic order pattern analysis for the separation of consciousness and unconsciousness: an analysis of approximate entropy, permutation entropy, recurrence rate, and phase coupling of order recurrence plots. Anesthesiology 109, 1014-1022 (2008).

88. Li, X., Cui, S. \& Voss, L. J. Using permutation entropy to measure the electroencephalographic effects of sevoflurane. Anesthesiology 109, 448-456 (2008)

89. Wilcox, R. R. \& Rousselet, G. A. A Guide to Robust Statistical Methods in Neuroscience. Curr. Protoc. Neurosci. 82, 8.42.1-8.42.30 (2018).

90. Pedregosa, F. et al. Scikit-learn: Machine Learning in Python. J. Mach. Learn. Res. 12, 2825-2830 (2011).

91. Ashburner, J. \& Friston, K. J. Unified segmentation. NeuroImage 26, 839-851 (2005).

92. Dmochowski, J. P. et al. Targeted transcranial direct current stimulation for rehabilitation after stroke. NeuroImage 75, 12-19 (2013).

93. Collignon, A. et al. Automated multi-modality image registration based on information theory. Bizais (1995).

94. Krähenbühl, P. \& Koltun, V. Efficient Inference in Fully Connected CRFs with Gaussian Edge Potentials. ArXiv12105644 Cs (2012).

95. Huang, Y. \& Parra, L. C. Fully automated whole-head segmentation with improved smoothness and continuity, with theory reviewed. PloS One 10, e0125477 (2015).

96. Huang, Y., Datta, A., Bikson, M. \& Parra, L. C. Realistic volumetric-approach to simulate transcranial electric stimulationROAST - a fully automated open-source pipeline. J. Neural Eng. 16, 056006 (2019).

97. Huang, Y. et al. Measurements and models of electric fields in the in vivo human brain during transcranial electric stimulation. eLife 6, e18834 (2017).

98. Fonov, V. et al. Unbiased average age-appropriate atlases for pediatric studies. NeuroImage 54, 313-327 (2011).

99. Fonov, V., Evans, A., McKinstry, R., Almli, C. \& Collins, D. Unbiased nonlinear average age-appropriate brain templates from birth to adulthood. NeuroImage 47, S102 (2009).

100. Ashburner, J. A fast diffeomorphic image registration algorithm. NeuroImage 38, 95-113 (2007).

101. Salter, K. C. \& Fawcett, R. F. The art test of interaction: a robust and powerful rank test of interaction in factorial models. Commun. Stat. - Simul. Comput. 22, 137-153 (1993).

102. Maris, E. \& Oostenveld, R. Nonparametric statistical testing of EEG- and MEG-data. J. Neurosci. Methods 164, 177-190 (2007).

103. Hedges, L. V. Distribution Theory for Glass's Estimator of Effect size and Related Estimators. J. Educ. Stat. 6, 107-128 (1981).

104. Cohen, J. Statistical Power Analysis for the Behavioral Sciences. (Routledge, 1988).

105. Oliphant, T. E. Python for Scientific Computing. Comput. Sci. Eng. 9, 10-20 (2007).

106. Wobbrock, J. O., Findlater, L., Gergle, D. \& Higgins, J. J. The Aligned Rank Transform for Nonparametric Factorial Analyses Using Only Anova Procedures. In Proceedings of the SIGCHI Conference on Human Factors in Computing Systems 143-146, https://doi. org/10.1145/1978942.1978963 (ACM, 2011).

107. Pernet, D. C. et al. Best Practices in Data Analysis and Sharing in Neuroimaging using MEEG, https://doi.org/10.31219/osf.io/ a8dhx (2018).

\section{Acknowledgements}

This work was supported by: 'e(ye)BRAIN, Ivry-sur-Seine, France', 'Institut National de la Santé et de la Recherche Médicale' (BH, LN, JS), Sorbonne Université (LN), the James S. McDonnell Foundation (LN), FRM 2015 (LN), UNIM (LN), Académie des Sciences-Lamonica Prize 2016 (LN). The research leading to these results has received funding from the program 'Investissements d'avenir' ANR-10- IAIHU-06, 'and by ANR grant 'CogniComa' (ANR-14-CE-0013-03). BH was funded by 'Poste d'Accueil Inserm' grant.

\section{Author contributions}

Study concept and design: B.H., B.R., J.D.S., F.F., F.R. and L.N. Data collection: S.D., F.F., B.H., P.P., B.R., M.V. and N.W. Analysis and interpretation of data: B.H., D.E., F.R., J.D.S., A.H., L.H., L.P. and L.N. Drafting of the manuscript: B.H. and L.N. Critical revision of the manuscript for important intellectual content: B.H., B.R., D.E., F.R., J.D.S., A.H., L.H., L.P. and L.N. Statistical analysis: B.H. and L.P. Study supervision: B.H., J.D.S. and L.N. had full access to all the data in the study and take responsibility for the integrity of the data and the accuracy of the data analysis. 


\section{Competing interests}

The authors declare no competing interests.

\section{Additional information}

Supplementary information is available for this paper at https://doi.org/10.1038/s41598-020-61180-2.

Correspondence and requests for materials should be addressed to J.D.S. or L.N.

Reprints and permissions information is available at www.nature.com/reprints.

Publisher's note Springer Nature remains neutral with regard to jurisdictional claims in published maps and institutional affiliations.

(c) (i) Open Access This article is licensed under a Creative Commons Attribution 4.0 International License, which permits use, sharing, adaptation, distribution and reproduction in any medium or format, as long as you give appropriate credit to the original author(s) and the source, provide a link to the Creative Commons license, and indicate if changes were made. The images or other third party material in this article are included in the article's Creative Commons license, unless indicated otherwise in a credit line to the material. If material is not included in the article's Creative Commons license and your intended use is not permitted by statutory regulation or exceeds the permitted use, you will need to obtain permission directly from the copyright holder. To view a copy of this license, visit http://creativecommons.org/licenses/by/4.0/.

(C) The Author(s) 2020 